Portland State University

PDXScholar

$1-1-2012$

\title{
Measurement and Simulation of Parallel Plate Waveguide Structures in the Terahertz Region for Sensing and Material Characterization Applications
}

James Alexander Higgins

Portland State University

Follow this and additional works at: https://pdxscholar.library.pdx.edu/open_access_etds Let us know how access to this document benefits you.

\section{Recommended Citation}

Higgins, James Alexander, "Measurement and Simulation of Parallel Plate Waveguide Structures in the Terahertz Region for Sensing and Material Characterization Applications" (2012). Dissertations and Theses. Paper 867.

https://doi.org/10.15760/etd.867

This Thesis is brought to you for free and open access. It has been accepted for inclusion in Dissertations and Theses by an authorized administrator of PDXScholar. Please contact us if we can make this document more accessible: pdxscholar@pdx.edu. 
Measurement and Simulation of Parallel Plate Waveguide Structures in the Terahertz Region for Sensing and Material Characterization Applications

\author{
by \\ James Alexander Higgins
}

A thesis submitted in partial fulfillment of the requirements for the degree of

\author{
Master of Science \\ in \\ Electrical and Computer Engineering
}

Thesis Committee:

Branimir Pejcinovic, Chair

Martin Siderius

Lisa Zurk

Portland State University

(c) 2012 
ABSTRACT

The Terahertz $(\mathrm{THz})$ region is a burgeoning field of research with applications in spectroscopy, integrated circuit fabrication, bio-medicine, and communications. Until recently, the $\mathrm{THz}$ region was largely unexplored, mainly due to the technical difficulties involved in making efficient and compact sources and detectors. As these challenges are addressed, the focus of research has shifted to practical applications, such as sensing and imaging.

The focus of this thesis is to investigate the characterization of parallel plate waveguide multimode propagation and periodically notched resonant structures for use in sensing and material parameter extraction applications. Broadband and narrowband measurements are presented and analyzed. Measurements are compared to finite difference time domain simulations and analytic solutions that use a Fourier transform mode-matching technique. Agreement is observed between simulation and measurement of radiation patterns. Weighted estimates of individual mode analytic solutions produce equivalent radiation patterns, which allows insight into the energy coupled into each respective mode. Results show that higher order modes contribute both a greater conductive attenuation and higher coupling loss. Agreement is also observed between measurements and simulated single and periodically notched resonant structures. Results demonstrate shifting of the resonant peak with respect to changes in plate separation for the periodically notched structure. For the single notch resonator, simulations indicate the resonant peak is dependent on notch depth until the depth-to-width ratio is greater than two.

This work demonstrates that multimode propagation can be identified and 
the amount of energy coupled into each mode may be estimated using radiation patterns. Experiments using quasi-optical time domain spectroscopic and continuous wave vector network analyzer systems in the $\mathrm{THz}$ region have been demonstrated. Finite difference time domain simulations have validated measurements on both systems. The results presented will advance the field of $\mathrm{THz}$ research by aiding in the design and analysis of sensing and material parameter extraction systems. 
DEDiCATION

To the moon of my life, Alicia Marie Smith. 


\section{ACKNOWLEDGEMENTS}

I would like to thank the following people, without their knowledge and support this body of work would not have been possible. My advisor Branimir Pejcinovic for continually challenging me and allowing me to explore all my intellectual curiosities, even when they appeared to be off task. My committee members, Martin Siderius and Lisa Zurk for their invaluable critiques and accommodating a rapid defense schedule. My colleagues in the Northwest Electromagnetics and Acoustics Research Laboratory and the Integrated Circuits Design and Test Laboratory for their engaging discusses and Friday afternoon luncheons. My research compeer Forest Kernan, it has been a delight and privilege working with you. Professors Donald Duncan and James E. Morris for inspiring and enhancing my academic experience. Mike Chuning the machinist extraordinaire, who taught me how use the equipment in the Mechanical and Materials Engineering machine shop and gave me design ideas for experiment fixtures and waveguides. My brother Timothy Higgins for reminding me that there is a light at the end of the tunnel, even when you can not longer see it. His wife Marisa for her grammatical expertise. My mother, father, and step-mother for their lifelong support and encouragement. Finally, I learned a great deal about myself professionally and personally through my experiences as a lab instructor and teaching assistant. So I would like to thank all the undergraduate students that I have worked with over these last few years; always question and keep pondering. 
TABle of Contents

Abstract

Dedication $\quad$ iii

Acknowledgements $\quad$ iv

List of Tables vii

List of Figures viii

List of Abbreviations $\quad$ xi

Preface xiii

1 Introduction 1

1.1 The Terahertz Gap . . . . . . . . . . . . . . . . . . . . 1

1.2 Present Applications of Terahertz Sensing and Material Characterization ......................... 4

1.3 Where This Work Contributes to the Field of Terahertz Spectroscopy 6

2 Background and Theory 11

2.1 Maxwell's Equations ..................... 11

2.2 Parallel Plate Waveguide . . . . . . . . . . . . . . . . . . . . 18

2.3 Fourier Transform Mode Matching Technique . . . . . . . . . . . . 23

3 Finite Difference Time Domain Simulation 32

3.1 MIT Electromagnetic Equation Propagation . . . . . . . . . . . . . 32

3.2 Gaussian Source Model . . . . . . . . . . . . . . . . . . . . . 39

4 Measurement Systems $\quad 42$

4.1 Time Domain Spectroscopy . . . . . . . . . . . . . . . . . . 42

4.2 Continuous-Wave Vector Network Analyzer . . . . . . . . . . . . . . 43

5 Results and Discussion $\quad 47$

5.1 Detection and Estimation of Parallel Plate Waveguide Multimode Operation from Radiation Patterns . . . . . . . . . . . . . . . . . 47

5.1.1 Experiment Setup . . . . . . . . . . . . . 49 
5.1.2 Radiation Pattern Experiment Alignment Process . . . . . . 53

5.1.3 Parallel Plate Waveguide Radiation Patterns . . . . . . . . . 54

5.1.4 Undefined Side Lobe Radiation Patterns . . . . . . . . . . . 59

5.2 Parallel Plate Waveguide Resonant Structures . . . . . . . . . . . . 65

5.2.1 Single Notch . . . . . . . . . . . . . . . . 65

5.2.2 Periodically Notched . . . . . . . . . . . . . . 68

6 Conclusion and Future Work 73

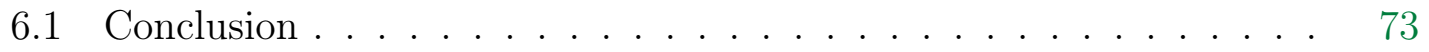

6.2 Future Work . . . . . . . . . . . . . . . . . . 77

$\begin{array}{lr}\text { References } & 80\end{array}$ 
LisT OF TABLES

1.1 Common units scaled to 1 THz. . . . . . . . . . . . . . 2

2.1 Differential and integral forms of Maxwell's equations. . . . . . . . . 12

2.2 Fundamental electromagnetic quantities. . . . . . . . . . . . . . 13

2.3 Flanged PPW TE mode cutoff frequencies for $d=0.5 \mathrm{~mm}$. . . . . 31

3.1 Scheme functions that convert international system of units (SI) values to scale-invariant units. . . . . . . . . . . . . . 36

4.1 Picometrix T-Ray ${ }^{\mathrm{TM}} 4000$ system specifications. . . . . . . . . . . . 42

4.2 Virginia Diodes Inc. (VDI) extender module specifications. . . . . . 44

5.1 First five parallel plate waveguide (PPW) cutoff frequencies calculated for transverse electric (TE) and transverse magnetic (TM) modes. (A) indicates mode cutoffs for a plate separation of $1.4 \mathrm{~mm}$ and (B) indicates mode cutoffs for a plate separation of $75 \mu \mathrm{m}$. . . . . . . . 48

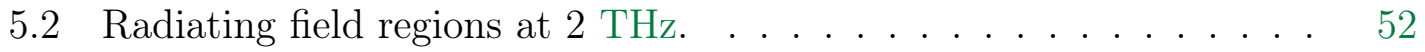

5.3 Resonant frequency values of simulated FDTD single notch depths. 68

5.4 Time Domain Spectroscopy (TDS) and Continuous wave Vector Network Analyzer (CW-VNA) system and finite difference time domain (FDTD) simulation resonant peak values for varying plate separation. 72 


\section{List OF Figures}

1.1 Electromagnetic spectrum indicating the "THz gap". . . . . . . . . . 2

1.2 Number of $\mathrm{THz}$ research related articles per year from $1985-2011 . \quad 3$

2.1 Diagram of the geometry for a PPW. . . . . . . . . . . . . . 19

2.2 Comparison of parallel plate waveguide TE and TM mode field strength distributions. ......................... 23

2.3 First three TE field distributions between the plates of the PPW. . 24

2.4 Cross section diagram of a radiation pattern geometry of a flanged PPW. . . . . . . . . . . . . . . 26

2.5 Contour path in the $\zeta$-plane. . . . . . . . . . . . . . . 28

2.6 Examples of PPW TE radiation patterns using (2.56). (A) indicates the first three even symmetric modes and (B) the odd. . . . . . . . 30

3.1 Example of FDTD grid spatial resolution required to represent a wave that will achieve second order accurate results. . . . . . . . . . . . 37

3.2 Multimode PPW resonator field distribution image created using h5utils (white regions are zero). . . . . . . . . . . . 38

3.3 Gaussian pulse model of the TDS system waveform compared to the FDTD source function. . . . . . . . . . . . . . . . 40

4.1 TDS system free space measurement. (A) Received waveform and (B) corresponding power spectrum. . . . . . . . . . . . . . . 43

4.2 Continuous wave Vector Network Analyzer system dynamic range for WR10 - WR1.5 bands. . . . . . . . . . . . . . . . . 45

5.1 Diagram indicating the PPW geometry and setup for a radiation pattern experiment. . . . . . . . . . . . . . 50

5.2 Pictures of the radiation pattern characterization experiments. (A) Shows the TDS system and (B) the CW-VNA system. . . . . . . . . 51

5.3 Radiation pattern experiment alignment process time response waveforms. (A) Misaligned TM mode and (B) aligned TE mode. . . . . . 53

5.4 Radiation pattern experiment alignment procedure diagram. . . . . 54 
5.5 Measured and simulated radiation pattern spectrograms of the PPW operating in the TE and TM mode. (A) and (B) are TDS system measurements. (C) and (D) are FDTD simulation results. (E) is CW-VNA system measurements. Note that CW-VNA measurements cover the 330 - $390 \mathrm{GHz}$ range, while TDS and FDTD results cover 100 - 1500 GHz. . . . . . . . . . . . . . . . . . . .

5.6 Example of the PPW multimode radiation pattern using weighted TE mode contributions of the analytic solution. . . . . . . . .

5.7 PPW TE multimode radiation pattern of FDTD simulation compared to weighted analytic mode contributions. . . . . . . . . .

5.8 PPW TE multimode radiation patterns of TDS and CW-VNA system measurements, and FDTD simulation compared to weighted analytic mode contributions. . . . . . . . . . . . . . . . . .

5.9 Theoretic conductive attenuation loss for even symmetric TE modes compared to TDS system measurements. . . . . . . . . . . . .

5.10 Measured TDS system excess conductive attenuation losses beyond the $\mathrm{TE}_{1}$ mode. Black points indicate radiation patterns with distinct side lobes. Red point indicates a radiation pattern without the expected side lobes. (A) Excess loss in TDS measurements and (B) the corresponding radiation patterns for indicated points. . . . . . . . .

5.11 Measured CW-VNA system excess conductive attenuation losses beyond the $\mathrm{TE}_{1}$ mode. Black points indicate radiation patterns with distinct side lobes. Red point indicates a radiation pattern without the expected side lobes. (A) Excess loss in CW-VNA measurements and (B) the corresponding radiation patterns for indicated points. .

5.12 TE multimode radiation patterns of FDTD and CW-VNA at 383 $\mathrm{GHz}$ compared to weighted analytic mode contributions. . . . . . .

5.13 TE multimode radiation patterns of FDTD and TDS at $910 \mathrm{GHz}$ compared to weighted analytic mode contributions. . . . . . . .

5.14 TDS radiation pattern measurements of a flanged PPW operating in TM multimode compared to FDTD simulations and theoretic weighted analytic solution. (A) Input frequency of $300 \mathrm{GHz}$ and (B) at $600 \mathrm{GHz} \ldots \ldots \ldots$. . . . . . . . . . . . . . . 65

5.15 Cross section diagram of the N-PPW resonant structure. . . . . . . 67

5.16 FDTD simulation results illustrating resonant peak shift for varying N-PPW depth-to-width ratios $(t / w) . \ldots . . . . . . . .$.

5.17 Cross section diagram of the notched periodic parallel plate waveguide (NP-PPW) resonant structure. . . . . . . . . . . . . . . 
5.18 Micrograph pictures of milled periodically notch PPW resonator. (A) Indicates the notches centered and milled across the width of the bottom plate of the NP-PPW, (B) a single notch width, (C) a single notch pitch, and (D) the center span length. . . . . . . . . . .

5.19 Comparison of TDS and CW-VNA system results and FDTD simulated transmission characteristics of the NP-PPW resonator. . . . . 
List of ABBreviations

VNA Vector Network Analyzer .......................... 44

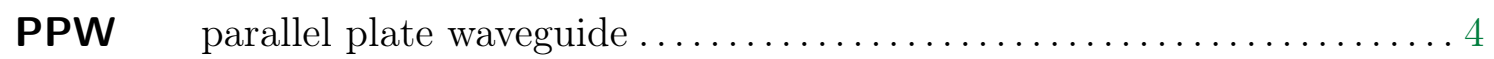

TEM transverse electromagnetic .......................... 17

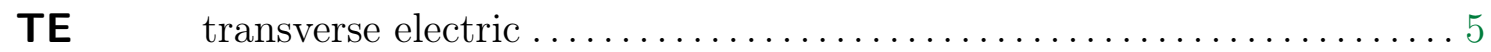

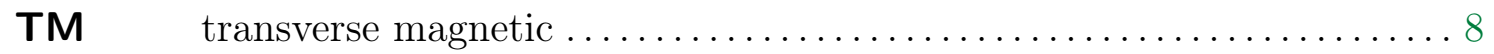

N-PPW notched parallel plate waveguide....................... 66

NP-PPW notched periodic parallel plate waveguide $\ldots \ldots \ldots \ldots \ldots \ldots \ldots \ldots 6$

TDS Time Domain Spectroscopy .........................

CW-VNA Continuous wave Vector Network Analyzer ................... 4

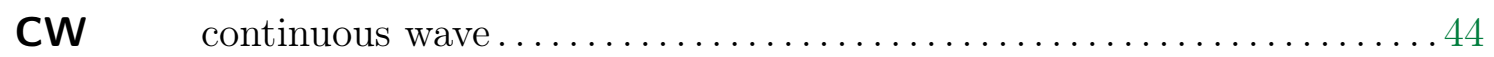

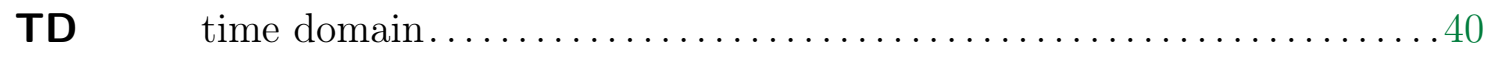

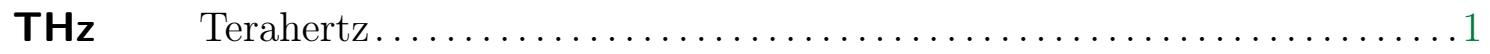

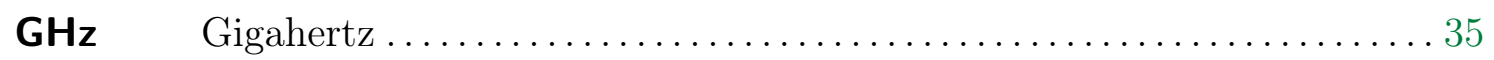

FDTD finite difference time domain .........................

FWHM full width half maximum ................................ 39

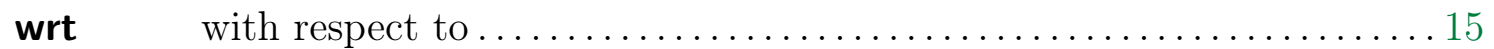

TOSM through open short match ........................... 50

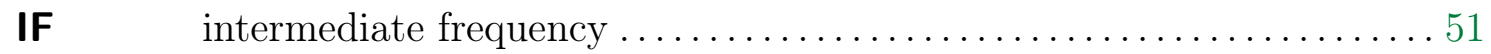

MEEP MIT electromagnetic equation propagation $\ldots \ldots \ldots \ldots \ldots \ldots \ldots \ldots$

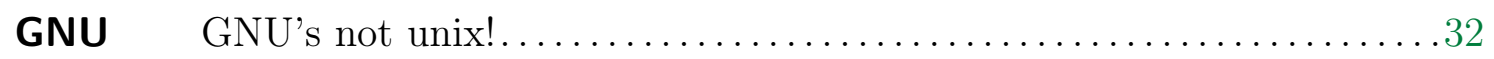

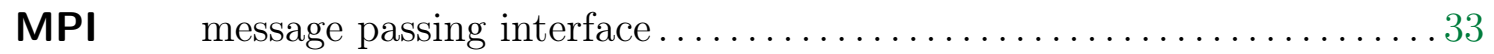

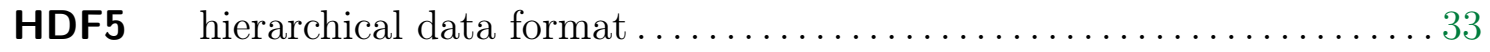

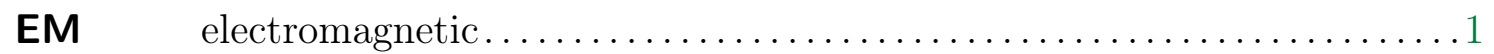

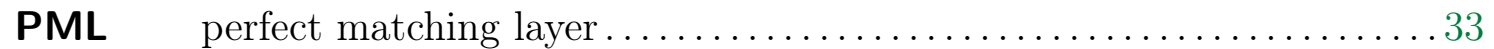

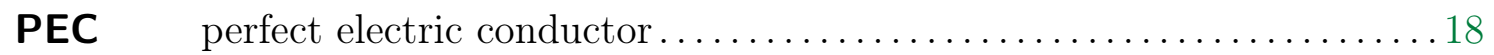

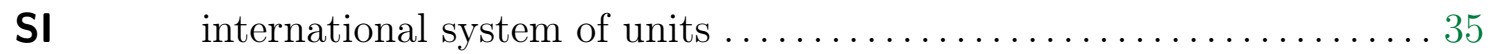

PNG portable network graphic ............................. 38 
ASCII American Standard Code for Information Interchange ............. 34

BRMSD brute-force recursive minimum standard deviation $\ldots \ldots \ldots \ldots \ldots \ldots$.

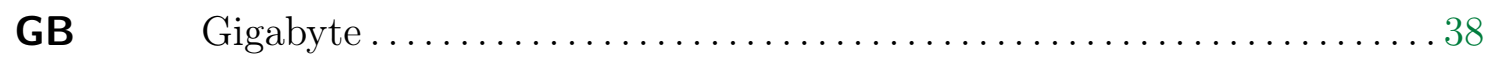

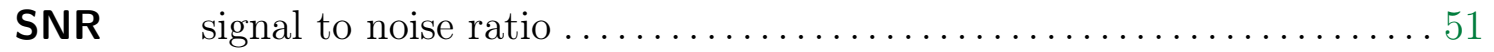

HDPE high-density polyethylene ........................... 43

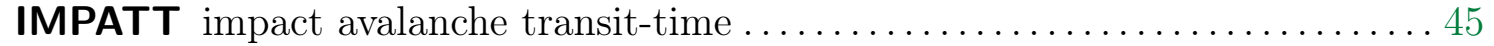

PC photoconductive ................................ 41

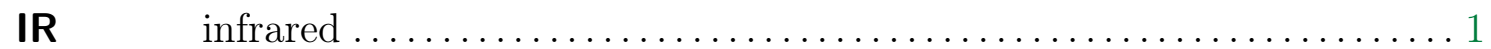

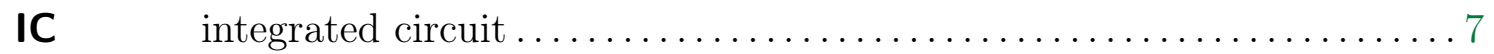

IEEE Institute of Electrical and Electronics Engineers ................ 2

LT-GaAs low temperature Gallium Arsenide

InP Indium Phosphide

Ge Germanium 
PREFACE

This document has been written with the intent that those with a general graduate level scientific background will be able to read it in its entirety and not go searching through lengthly lists of references for explanations. As such, there is a lot of background material presented and care is taken to explicitly describe the theories which this work relies on. Additionally, or perhaps consequently, the very nature of research work is to push the limits of what is known and explore what is unknown. With that stated, for the sake of brevity I have omitted lengthly derivations that detract from the understanding of a theory and simply cited the end result and its source. Like most research work there are artifacts in the results which are not well understood at this time and merit further investigation. When this situation is encountered, I have offered educated hypotheses as to their origins. Maybe one of these situations will peak your curiosity, the way it did mine, or maybe you already know its origins. Either way, it is my hope that by reading this work you too will be inspired to expand your knowledge and understanding of the world in which we live. 
CHAPTER 1

INTRODUCTION

\subsection{The Terahertz GaP}

Terahertz $(\mathrm{THz})$ radiation is electromagnetic (EM) radiation with energy associated with frequencies which lie between microwave and infrared regions in the spectrum. Technological difficulties involved with creating efficient and compact sources and detectors for this region left it largely unexplored. This led to it being colloquially referred to as the "THz gap". Although, many of the challenges involved with the generation and detection of $\mathrm{THz}$ radiation have been addressed and research in this region is now actively being pursued. While the $\mathrm{THz}$ band still does not have a standard definition, research in the spectrum from 0.1 to $30 \mathrm{THz}$ is commonly

considered $\mathrm{THz}\left(10^{12} \mathrm{~Hz}\right)$. The lower limit of this region covers millimeter (1-10 $\mathrm{mm})$ and sub-millimeter $(0.1-1 \mathrm{~mm})$ waves and extends into the far-infrared (IR) (200-350 $\mu \mathrm{m}$ ) band. The EM spectrum indicating the extent of the "THz gap" is shown in Figure 1.1. Commonly used units and their conversion at $1 \mathrm{THz}$ is shown in Table 1.1, where $c=3 \times 10^{8}(\mathrm{~m} / \mathrm{s})$ is the vacuum speed of light and $h=4.136$ $(\mathrm{eV} \cdot \mathrm{s})$ is Plank's constant. 


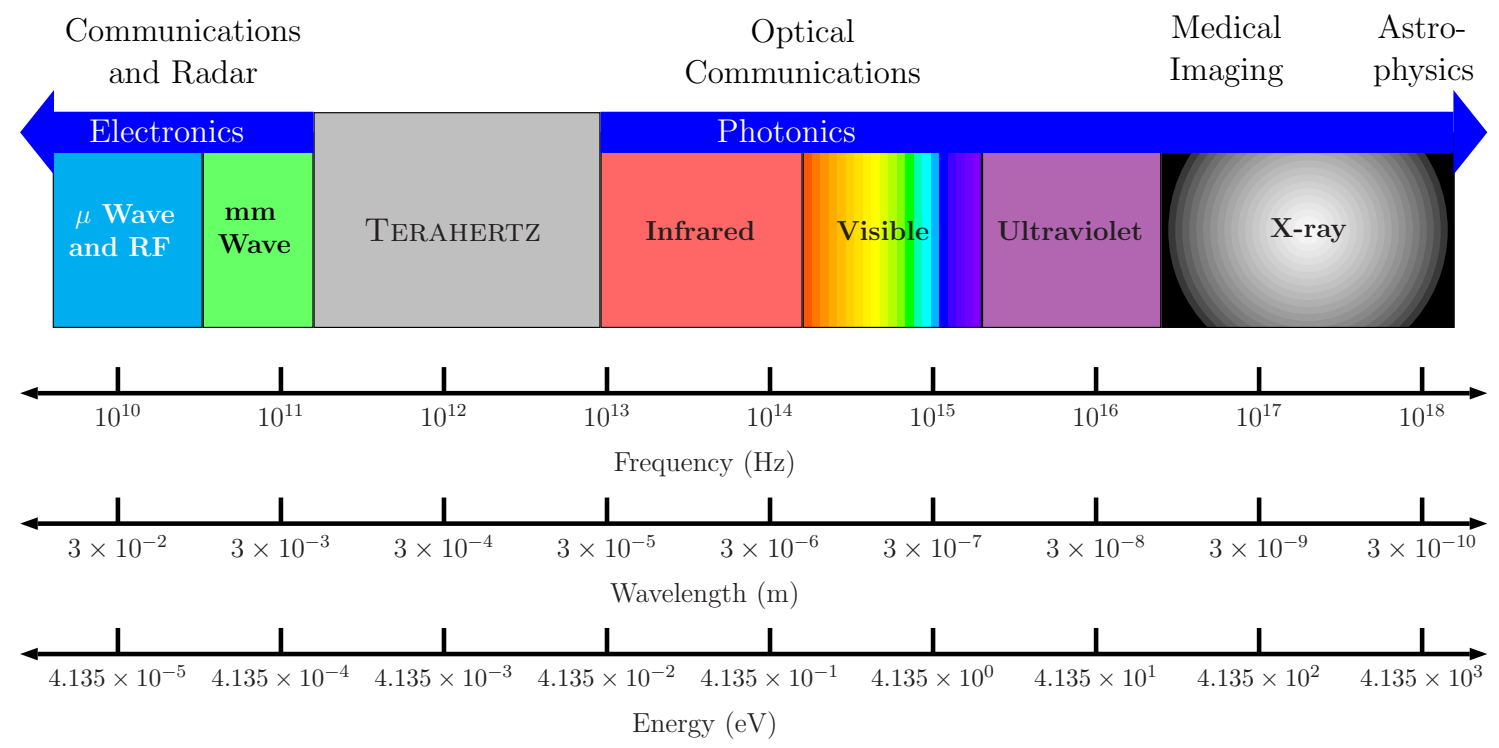

FigURE 1.1 - Electromagnetic spectrum indicating the "THz gap".

$\mathrm{THz}$ research has been a growing area for the last 25 years, as shown in Figure 1.2. Data for the histogram shown in Figure 1.2 was collected from the http://www.engineeringvillage2.org website. This search was performed Dec. 14th, 2011 for all articles containing either "Terahertz" or "THz" in their Subject/Title/Abstract from 1984 to present. A decline in the published research work in this region over the most recent two years indicates that the field is reaching its maturity. Another indication of this is the newly created Institute of Electrical and Electronics Engineers (IEEE) Transactions on Terahertz Science and Technology. This journal brings together the very broad range of topics and technologies that

\begin{tabular}{|c|c|}
\hline Unit & Conversion at $\mathbf{1} \mathbf{~ T H z}$ \\
\hline Frequency $\nu$ & $\nu=1 \mathrm{THz}=1000 \mathrm{GHz}$ \\
\hline Wavelength $\lambda$ & $\lambda=\frac{c}{\nu}=300 \mu \mathrm{m}$ \\
\hline Photon Energy $\mathrm{E}$ & $\mathrm{E}=h \nu=4.14 \mathrm{meV}$ \\
\hline
\end{tabular}

TABLE 1.1 - Common units scaled to $1 \mathrm{THz}$. 
encompass $\mathrm{THz}$ techniques and applications. As this area of study matures its focus of research has shifted to practical applications, such as sensing, imaging, and material characterization. These practical applications rely on spectroscopic techniques, where the spectral signature of a material is based on its ability to absorb only energy of specific frequencies. Many biomolecules and explosive compounds absorb radiation in the $\mathrm{THz}$ region, which allows them to be identified using broadband THz spectroscopy. Additional, common materials like paper products, plastics, and clothing fibers are virtually transparent to $\mathrm{THz}$ radiation. This combined with the fact that $\mathrm{THz}$ radiation is non-ionizing makes it well suited for health safety scanning of foods and transportation security.

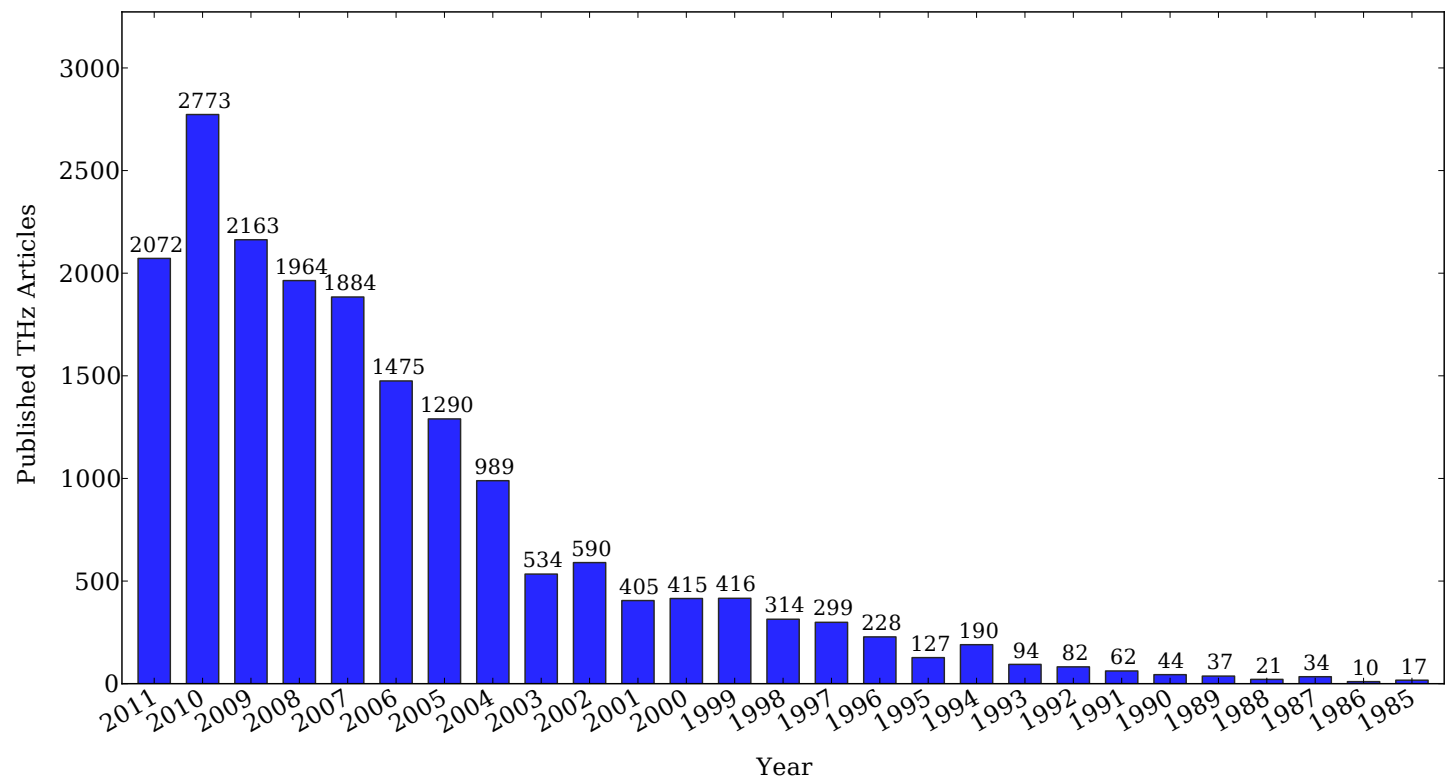

FigURE 1.2 - Number of THz research related articles per year from 1985 - 2011. 


\subsection{Present Applications of Terahertz Sensing and Material Char- ACTERIZATION}

The parallel plate waveguide (PPW) remains a subject of particular interest in the field of $\mathrm{THz}$ sensing and material parameter extraction due to the ease in which it can be manufactured, its well defined theory, and its extended material-EM field interactions. Recently, a comprehensive study of the $\mathrm{TE}_{1}$ dominant mode of propagation suggests that it may be best suited for sensing applications [1]. Although, relatively little work has been done in characterizing PPW operating in a multimode configuration. This is mainly due to unformalized mode excitation and conversion loss theory. Understanding multimode operation is especially important when broadband spectrographic systems are utilized, where multimode operation is inevitable because the broad frequency content of the pulsed system couples energy into higher order modes.

The typical design decision for maintaining dominant mode of operation over the desired measurement band is to modify the geometry of the waveguide. In the case of the PPW, this is achieved by decreasing the plate separation, which increases the dominant mode cutoff frequency and widens the mode bandwidth. Using this methodology requires multiple waveguides with subsequently smaller dimensions to cover the entire $\mathrm{THz}$ region; in fact, this is what the Continuous wave Vector Network Analyzer (CW-VNA) system implements. However, reducing the dimensions of the waveguide introduces propagation loss, group velocity dispersion, and coupling issues [2]. Mode-matching techniques [3] have been developed to optimize the PPW input coupling to selectively excite only the dominant mode, but again, these techniques rely heavily on spatial scaling not only of the incident beam but also the 
geometry of the PPW. By characterizing the behavior of the PPW operating in a multimode configuration corrections may be applied to subsequent measurements which would relax the source and waveguide dimension restrictions that single mode techniques require.

This work investigates the flanged PPW operating in a transverse electric (TE) multimode configuration. Measurements presented are taken with broadband Time Domain Spectroscopy (TDS) and narrowband CW-VNA systems. These measurements are compared to analytic solutions using a Fourier transform modematching technique [4] and computer simulation using a finite difference time domain (FDTD) technique [5]. The flanged PPW was selected for this investigation since multiple numeric approaches and an analytic series solution exist. The analytic series solution that was used reports the calculated reflection coefficient over a range of waveguide opening to wavelength ratios. It is important to note that the analytic series solution is input independent. Therefore, this solution may be used to characterize multimode behavior regardless of input coupling, as long as the output is flanged. A boundary-element method has been reported [6] for the case of an arbitrarily flanged PPW. Their findings suggest that other geometries are better suited for directionality and side lobe suppression. Unfortunately, the method reported in [6] does not lend itself to multimode characterization, but the analytic series solution based on the Fourier transform mode-matching technique does.

The efficient detection of biomolecules is increasingly important in the biological, medical, and pharmaceutical industries. Standard techniques often include costly and cumbersome marking processes to identify biomolecules. THz spectroscopy is well suited as an alternative since many biomolecules have spectral signatures in this region of the EM spectrum. Important attributes of a biosensor system 
are performance and cost efficiency. The integration of system components on chip serves to increase performance and reduce overall system size. However, these systems typically use semiconductors (i.e. LT-GaAs, InP) with high electron mobilities for photoconductive switches that generate and detect THz signals. The fabrication processes for these devices are expensive and ill suited for biological applications that require disposable sensors. In contrast, the transmission lines (waveguides) and biosensing resonant structures are passive components, which are inexpensive to fabricate. By separating the expensive active components from the cheaper passive components a modular biosensor system with cheap disposable sensors is realized.

In the following section, single notch and periodically notched PPW resonant structures are investigated for use as $\mathrm{THz}$ biosensors. To date, there are no known works that link the characteristic design parameters of these structures to their peak resonance. Field solutions for similar structures has been presented [7, 8]. These solutions lend themselves to computationally efficient close-form expressions for the structure's transmitted power. However, these analytic solutions only cover simple rectilinear geometries. This leaves the typical design process for these devices to iterative EM simulations where design parameters are refined to meet their respective specification. This work focuses on characterizing the effects of two major design parameters: notch depth and plate separation.

\subsection{Where This Work Contributes to the Field of Terahertz Spec- TROSCOPY}

The efficient use of $\mathrm{THz}$ radiation for spectroscopy is increasingly important in the biomedicine, security, and semiconductor industries. The biomedicine and security 
industries are interested in $\mathrm{THz}$ spectroscopy because many biomolecules and explosives have unique spectral signatures in this region of the EM spectrum. The semiconductor industry is interested in $\mathrm{THz}$ spectroscopy in order to characterize current integrated circuit (IC) device fabrication materials. Typical spectroscopic measurements expose a sample to $\mathrm{THz}$ radiation in free space, simply because this is the easiest experiment to perform. However, free-space interaction between $\mathrm{THz}$ radiation and samples has many problems, e.g. water vapor absorption, short interaction times, and surface roughness. If reflected waves are utilized instead of transmitted waves, additional problems arise, as discussed in [9]. In many cases it is advantageous to use some form of waveguide to direct the radiation from the transmitter to the sample and on to the receiver.

The PPW is a structure well suited for both biomedicine and security sensing and semiconductor material parameter extraction. False sensor readings can be diminished by designing PPW resonant structures that are tuned to detect only one material's unique spectral signature and by placing samples laterally inside the PPW. This way the material-EM field interactions occur over longer distances than in free space. But, the use of PPW for broadband spectroscopic applications raises some interesting engineering challenges of its own. First, waveguide theory introduces multiple modes of operation which lead to excess energy loss in dispersion and mode conversion. Second, resonance of the notched PPW structure is dependent on its geometric parameters; plate separation, notch width, depth, pitch, and center spacing. The plate separation can be adjusted (tuned) in-field, but the notch geometries and their placement must be determined prior to fabrication. This work addresses these issues by identifying and characterizing the behavior of PPW operating in a multimode configuration and investigating the sensitivity of the geometric 
parameters of PPW resonant structures.

Over the course of this work $\mathrm{THz}$ experiments were developed for PPW radiation pattern characterization and measuring resonant structures. Custom PPWs and fixtures were designed and fabricated for each experiment. This PPW design included a crossed screw and positioning pin system that insured alignment of the top and bottom plates and a way to control and maintain the plate separation. Measurements were performed and presented on both broadband TDS and narrowband CW-VNA systems. A configurable Matlab package was developed to process experimental results. This package includes: utilities to rapidly parse data file formats from TDS and CW-VNA systems and FDTD simulation data, signal processing utilities to Fourier transforms with windowing and zero-padding, and utilities for quick plotting and figure exporting in uniform styles. An analytic solution for the radiation pattern of a flanged PPW was implemented in MATLAB. This implementation used the Fourier transform mode-matching technique outlined by Eom and Park $[4,10]$ and operates in TE and transverse magnetic (TM) mode. A brute-force recursive minimum standard deviation (BRMSD) algorithm was implemented in MATLAB to estimate the energy coupling into each mode. This algorithm estimates the energy coupled into each mode by minimizing the standard deviation between a radiation pattern composed of the weighted sum of theoretic modes and measured results. FDTD simulations were developed and performed for PPW radiation patterns and resonant structures. Macintosh ports were written for the freely available MIT electromagnetic equation propagation (MEEP) package [5] for EM simulation.

Work from this thesis regarding PPW radiation pattern characterization was published in the 76th ARFTG conference proceedings [11]. Additionally, part 
of the work included in this thesis regarding the FDTD simulations has been published in 34th MIPRO conference proceedings [12]. Other works that have indirectly contributed to these findings as a whole include: an investigation into $\mathrm{PPW} \mathrm{THz}$ radiation input coupling [13], utilizing PPW mode for THz sensing [14], and a characterization of Zinc Oxide nanowires using PPW [15].

The contributing work discussed in this section is summarized in the following list:

- Measurements were performed and presented on both broadband TDS and narrowband CW-VNA systems.

- Custom PPW and fixtures were designed and fabricated for each experiment.

- A configurable Matlab package was developed to process experimental results.

- An analytic solution for the radiation pattern of a flanged PPW [4, 10] was implemented in MATLAB .

- A BRMSD algorithm was implemented in MATLAB to estimate the energy coupled into each PPW mode.

- FDTD simulations were developed and performed for PPW radiation patterns and resonant structures.

- Macintosh ports were written for the freely available MEEP package [5] for EM simulation.

- Published works:

- 2011 SPIE Defense, Security, and Sensing conference [13]

- 76th ARFTG conference [11]

- 34th MIPRO conference [12]

- 11th IEEE-NANO conference [15] 
- 35th IRMMW-THz conference [14] 
Chapter 2

BACKGROUND AND THEORY

\subsection{MAXWELL'S EQUATIONS}

Electric and magnetic wave phenomena at the macroscopic level are described by Maxwell's equations, as published by James Clerk Maxwell in 1873 [16]. His work was based on the body of empirical and theoretical findings of Gauss, Ampere, Faraday, Kelvin, and others. It summarized the contemporaneous state of electromagnetic science and hypothesized the existence of the electric displacement current. At the time, Maxwell's postulate had little immediate impact, partly because scholars referred to his work as "lacking clear focus" and "notoriously hard to read" [17]. Surprisingly, his original work doesn't contain the now famous "Maxwell's equations" or mention the generation or detection of EM waves. It wasn't until after Maxwell's death that work by FitzGerald, Lodge, and Heaviside formalized what we now refer to as Maxwell's equations [17]. While the implications of Maxwell's work were not prevalent during his lifetime, the addition of the electric displacement current to Ampere's circuital law allowed him to join the physical laws of electricity and magnetism into one single unified theory. The unification of these theories explains many phenomena, not the least of which is that it allows the description of EM waves.

This section outlines the fundamental concepts of EM theory that are needed for the rest of this work. Maxwell's equations are presented, then wave propagation, and boundary conditions. Next, guided waves and the operating modes of PPW 
are discussed. Finally, the Fourier transform mode-matching technique for solving scattering and boundary value problems is presented.

\begin{tabular}{|c|c|c|}
\hline & Differential Form & Integral Form \\
\hline Faraday' law & $\nabla \times \overrightarrow{\mathcal{E}}=-\frac{\delta \overrightarrow{\mathcal{B}}}{\delta t}-\overrightarrow{\mathcal{M}}$ & $\oint \overrightarrow{\mathcal{E}} \cdot d \vec{l}=-\frac{\delta}{\delta t} \int_{S} \overrightarrow{\mathcal{B}} \cdot d \vec{s}-\int_{S} \overrightarrow{\mathcal{M}} \cdot d \vec{s}$ \\
\hline Ampere's law & $\nabla \times \overrightarrow{\mathcal{H}}=\frac{\delta \overrightarrow{\mathcal{D}}}{\delta t}+\overrightarrow{\mathcal{J}}$ & $\oint \overrightarrow{\mathcal{H}} \cdot d \vec{l}=\frac{\delta}{\delta t} \int_{S} \overrightarrow{\mathcal{D}} \cdot d \vec{s}+\int_{S} \overrightarrow{\mathcal{J}} \cdot d \vec{s}$ \\
\hline Gauss's law & $\nabla \cdot \overrightarrow{\mathcal{D}}=\rho$ & $\int_{S} \overrightarrow{\mathcal{D}} \cdot d \vec{s}=\mathcal{Q}$ \\
\hline (no name) & $\nabla \cdot \overrightarrow{\mathcal{B}}=0$ & $\int_{S} \overrightarrow{\mathcal{B}} \cdot d \vec{s}=0$ \\
\hline
\end{tabular}

TABLE 2.1 - Differential and integral forms of Maxwell's equations.

Plane waves are the simplest form of EM waves. Their basic properties and wave propagation serve to illustrate Maxwell's equations. Detailed derivations of Maxwell's equations is found in most EM texts [18, 19, 20, 21, 22]. The material presented in the following sections has been reinterpreted from these texts. Maxwell's equations in differential and integral form are summarized in Table 2.1. The script quantities in Table 2.1 indicate time-varying vector fields and are real valued functions of the independent spatial coordinates $x, y, z$ and temporal variable t. The fundamental EM quantities are defined in Table 2.2.

The sources for the EM field are the currents $\overrightarrow{\mathcal{M}}$ and $\overrightarrow{\mathcal{J}}$, and $\rho$ the electric charge density. All physical currents are the product of moving electric charge. In the case of the magnetic current, this would be a loop of electric current or a similar type of magnetic dipole. Magnetic monopole charges are not known to physically exist. Therefore, the magnetic current density $\overrightarrow{\mathcal{M}}$ is a fictitious source used for 


\begin{tabular}{|c|c|c|}
\hline Quantity & Physical Description & (MKS) Unit \\
\hline $\overrightarrow{\mathcal{E}}$ & electric field intensity & $\mathrm{V} / \mathrm{m}$ \\
\hline $\overrightarrow{\mathcal{H}}$ & magnetic field intensity & $\mathrm{A} / \mathrm{m}$ \\
\hline $\overrightarrow{\mathcal{D}}$ & electric flux density & $\mathrm{V} / \mathrm{m}^{2}$ \\
\hline $\overrightarrow{\mathcal{B}}$ & magnetic flux density & $\mathrm{Wb} / \mathrm{m}^{2}$ \\
\hline $\overrightarrow{\mathcal{M}}$ & magnetic current density & $\mathrm{V} / \mathrm{m}^{2}$ \\
\hline $\overrightarrow{\mathcal{J}}$ & electric current density & $\mathrm{A} / \mathrm{m}^{2}$ \\
\hline$\rho$ & electric charge density & Coul $/ \mathrm{m}^{2}$ \\
\hline $\mathcal{Q}$ & total charge & Coul \\
\hline
\end{tabular}

TABLE 2.2 - Fundamental electromagnetic quantities.

mathematical convenience. The amount of electric charge flow depends on electric charge density $\rho$, so it can be argued that $\rho$ is the true source of an electromagnetic field.

The following relations hold between the electric and magnetic fields and their respective flux densities:

$$
\begin{aligned}
& \overrightarrow{\mathcal{B}}=\mu \overrightarrow{\mathcal{H}}, \\
& \overrightarrow{\mathcal{D}}=\epsilon \overrightarrow{\mathcal{E}}
\end{aligned}
$$

where $\mu(\mathrm{H} / \mathrm{m})$ is the permeability of propagating medium, and $\epsilon(\mathrm{F} / \mathrm{m})$ is the permittivity of the propagating medium. A medium's electromagnetic properties are related to their respective free-space values by $\mu_{0}=\frac{\mu}{\mu_{r}}=4 \pi \times 10^{-7} \mathrm{H} / \mathrm{m}$ and $\epsilon_{0}=\frac{\epsilon}{\epsilon_{r}}=\frac{1}{36 \pi} \times 10^{-12} \mathrm{~F} / \mathrm{m}$, where the $r$ subscript indicates the medium's relative permeability and permittivity, respectively.

As stated, Maxwell's equations are valid for an arbitrary time dependence. In this work the fields involved have a sinusoidal time dependence with steady 
state conditions assumed. Therefore, using time-harmonic notation is convenient. If all field quantities are assumed to be complex vectors with an implied $e^{j \omega t}$ time dependence, then a time-harmonic electric field has the form

$$
\begin{aligned}
\overrightarrow{\mathcal{E}}(x, y, z ; t) & =\hat{u} A(x, y, z) \cos (\omega t+\phi) \\
& =\operatorname{Re}\left[\vec{E}(x, y, z) e^{j \omega t}\right] \\
\vec{E}(x, y, z) & =\hat{u} A(x, y, z) e^{j \phi}
\end{aligned}
$$

where $A$ is a real amplitude, $\omega$ is the angular frequency, and $\phi$ is the phase reference. Changing Maxwell's equations to their respective time-harmonic form involves converting each field quantity and replacing the time derivatives in Table 2.1 with $j \omega$. Maxwell's equations in time-harmonic form are shown in (2.1) - (2.4). Throughout this work the Fourier transform is used to convert an arbitrary time-dependent field to its time-harmonic representation

$$
\begin{aligned}
\nabla \times \vec{E} & =-j \omega \vec{B}-\vec{M}, \\
\nabla \times \vec{H} & =j \omega \vec{D}+\vec{J}, \\
\nabla \cdot \vec{D} & =\rho, \\
\nabla \cdot \vec{B} & =0 .
\end{aligned}
$$

\section{Plane-Wave Propagation}

The description of a traveling wave is possible using Maxwell's curl equations. In a source-free, linear, isotropic, homogeneous region the EM wave equations (2.5) and 
(2.6) take the form of the Helmholtz equation, where the constant $k=\omega \sqrt{\mu \epsilon}$ is called the wavenumber or propagation constant.

$$
\begin{aligned}
\nabla^{2} \vec{E}+k^{2} \vec{E} & =0 \\
\nabla^{2} \vec{H}+k^{2} \vec{H} & =0
\end{aligned}
$$

In lossless media the permittivity $\epsilon$ and permeability $\mu$ are real, so the wavenumber $k$ is also real. A general solution for each of the field components of (2.5) or (2.6) is obtained by the separation of variables method. Since each individual component is of the same form, only one needs to be obtained and the others can be determined by inspection. Consider an electric field with only a $\hat{x}$ component, then (2.5) can be solved to produce the traveling wave equation

$$
\begin{aligned}
E_{x}(z) & =A^{+} e^{-j k z}+A^{-} e^{j k z}, \\
\mathcal{E}_{x}(t, z) & =A^{+} \cos (\omega t-k z)+A^{-} \cos (\omega t+k z),
\end{aligned}
$$

where $A^{+}$and $A^{-}$denote arbitrary amplitudes with the forward (positive) and reverse (negative) propagating wave. This is more easily seen by converting (2.7) to its time dependent form (2.8). In order to maintain a fixed point on the wave, the argument (phase) of the cosine must remain constant. In this sense, the velocity of the wave is called the phase velocity $v_{p}$ because it maintains a fixed phase point on the wave regardless of propagation. Classically, the velocity is the distance traveled over a given time, $\Delta z / \Delta t$. By solving the argument of the cosine for $z$ and substituting the result into this differential with respect to (wrt) time, the relationship 
between the phase velocity and the propagating medium is determined as

$$
v_{p}=\frac{d z}{d t}=\frac{d}{d t}\left(\frac{\omega t-K}{k}\right)=\frac{\omega}{k}=\frac{1}{\sqrt{\mu \epsilon}}=\frac{c}{\sqrt{\mu_{r} \epsilon_{r}}}
$$

where $K$ is arbitrary constant and $c$ is the vacuum speed of light. Waves that have displacement orthogonal to their direction of propagation are called transverse waves. Two fundamental parameters of such waves are the wavelength $\lambda$ and period T. They are defined as the spatial, or temporal separation (parallel to the direction of propagation), between repetitions in the wave shape in which the displacement is the same. This is formalized by

$$
\begin{aligned}
(\omega t-k z)-(\omega t-k(z+\lambda)) & =2 \pi, \\
\lambda & =\frac{2 \pi}{k},
\end{aligned}
$$

and

$$
\begin{aligned}
(\omega t-k z)-(\omega(t+T)-k z) & =2 \pi \\
T & =\frac{2 \pi}{\omega} .
\end{aligned}
$$

Substituting these parameters into (2.9) yields

$$
v_{p}=\frac{\lambda}{T}=\lambda \nu
$$

which indicates that speed of the wave is one wavelength per oscillation. A complete description of the plane wave EM field must include the magnetic field. In general, if one quantity is known the other can be determined using Maxwell's curl equations. 
Applying (2.2) to (2.7) gives the magnetic field of

$$
H_{y}=\sqrt{\frac{\epsilon}{\mu}}\left(A^{+} e^{-j k z}+A^{-} e^{j k z}\right) .
$$

Note that $\vec{E}$ and $\vec{H}$ are orthogonal to the direction of propagation $( \pm \hat{z})$. Traveling waves of this type are by definition transverse electromagnetic (TEM) waves.

If the medium in which the waves propagate has conductive losses, then the propagation constant is considered to be complex. Substituting Ohm's law for conduction current into (2.2) yields

$$
\begin{aligned}
\nabla \times \vec{E} & =-j \omega \mu \vec{H}, \\
\nabla \times \vec{H} & =j \omega \epsilon \vec{E}+\sigma \vec{E},
\end{aligned}
$$

where $\sigma$ is the conductivity of the medium, $\sigma \vec{E}$ is the conduction current, and $j \omega \epsilon \vec{E}$ is the displacement current. The resulting wave equation for $\vec{E}$ becomes

$$
\nabla^{2} \vec{E}+\omega^{2} \mu \epsilon\left(1-j \frac{\sigma}{\omega \epsilon}\right) \vec{E}=0
$$

Comparing this to the lossless case, the complex propagation constant for the medium is given by

$$
\gamma=\alpha+j \beta=j \omega \sqrt{\mu \epsilon} \sqrt{1-j \frac{\sigma}{\omega \epsilon}} .
$$

Substituting (2.17) into 2.7 yields another traveling wave equation

$$
E_{x}(z)=A^{+} e^{-\alpha z}\left(e^{-j k z}+A^{-} e^{j k z}\right)
$$

this time the amplitude diminishes by an exponential damping factor. The rate of 
decay wrt distance is called the attenuation constant $\alpha$.

\section{BOUNDARY CONDITIONS}

In the preceding discussion plane-waves propagated in the same medium. The question now is: what happens to the fields at the interface of two media. Continuity of the fields at the interface dictates that their normal and tangential components are equal. In the case of the perfect conductor $(\sigma \rightarrow \infty)$ the tangential electric field components must be zero inside the conducting region. Applying the criteria for continuity of the fields to $(2.1)-(2.4)$ yields:

$$
\begin{aligned}
& \hat{n} \cdot \epsilon \vec{E}=\rho_{s}, \\
& \hat{n} \cdot \mu \vec{H}=0, \\
& \hat{n} \times \vec{E}=0, \\
& \hat{n} \times \vec{H}=\vec{J}_{s} .
\end{aligned}
$$

\subsection{Parallel Plate Waveguide}

The PPW is the simplest guide wave structure. In this work it is characterized by conductor boundaries that are parallel to the z-axis. For its analysis it is assumed to be uniform in the $z$ direction. The geometry of the PPW is shown in Figure 2.1, where perfect electric conductor (PEC), $d$ is the plate separation, $W$ is the width, and $l$ is the length. Note that, while not displayed to scale, $W$ and $l$ are assumed to be much greater than $d$. A material fills the region between the plates, in this case free space $\left(\mu_{r}=\epsilon_{r}=1\right)$.

The procedure for analyzing waveguides is the following: 


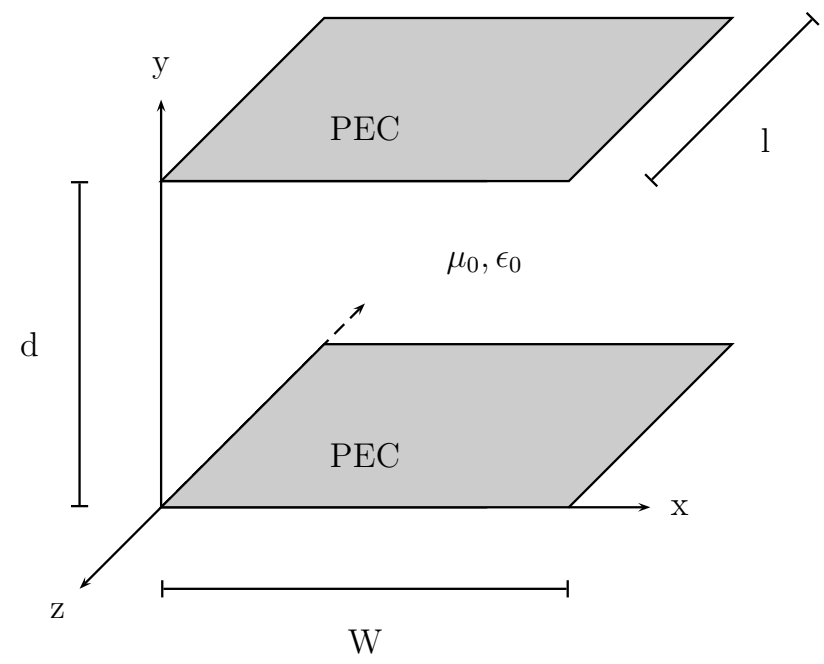

FIGURE 2.1 - Diagram of the geometry for a PPW.

1. Solve the 2D Helmholtz equation for the longitudinal $E$ or $H$ field component. This solution will contain the unknown cutoff wavenumber $k_{c}$ and several unknown constants.

2. Solve Maxwell's to find the transverse fields from the longitudinal $E$ or $H$.

3. Apply boundary conditions to the appropriate field components to determine the unknown constants and $k_{c}$.

4. Determine the propagation constant.

\section{Transverse Electric Modes}

TE modes are defined by $E_{z}=0$. Applying the procedure outlined prior, $\partial / \partial x=0$ and $H_{z}$ must satisfy the $2 \mathrm{D}$ wave equation

$$
\left(\frac{\partial^{2}}{\partial y^{2}}+k_{c}^{2}\right) h_{z}(x, y) e^{-j \beta z}=0
$$


where $k_{c}=\sqrt{k^{2}+\beta}$. A general solution to $(2.20)$ is

$$
h_{z}(x, y)=A \sin k_{c} y+B \cos k_{c} y \text {. }
$$

The boundary conditions are that $E_{x}=0$ at $y=\{0, d\}$. This implies that the coefficient of the sine function is zero $(A=0)$. Substituting (2.20) into (2.1) yields

$$
E_{x}=-j \frac{\omega \mu}{k_{c}^{2}} \frac{\partial}{\partial y} H_{z}=-j \frac{\omega \mu}{k_{c}^{2}}\left(A \cos k_{c} y-B \sin k_{c} y\right)
$$

Applying the boundary condition that $A=0$ and $k_{c}=m \pi / d$, where $m=\{(2 n+$ 1) $\} \mid n \in \mathbb{N}_{0}$ yields

$$
H_{z}=B_{m} \cos \left(\frac{m \pi}{d} y\right) e^{-j \beta z}
$$

From $H_{z}$ the transverse fields can be determined as

$$
\begin{aligned}
E_{x} & =j \frac{\omega \mu}{k_{c}^{2}} B_{m} \sin \left(\frac{m \pi}{d} y\right) e^{-j \beta z} \\
H_{y} & =j \frac{\beta}{k_{c}^{2}} B_{m} \sin \left(\frac{m \pi}{d} y\right) e^{-j \beta z} \\
E_{y} & =H_{x}=0
\end{aligned}
$$

Where the bounded propagation constant $\beta$ is

$$
\beta=\sqrt{k^{2}-k_{c}^{2}}=\sqrt{k^{2}-\left(\frac{m \pi}{d}\right)^{2}} .
$$

The sources of energy loss in this system are from dielectric and conductor. Since the waveguide under analysis is filled by free space, dielectric losses are zero. Energy attributed to conductor loss is associated with the energy dissipated in 
the conductor upon subsequent reflections as the wave propagates down the guide. This type of loss can be determined by using the perturbation method or Wheelers incremental inductance rule $[18,20]$. Attenuation due to conductor loss is

$$
\alpha_{c}=\frac{P_{l}}{2 P_{0}}=\frac{2 k_{c}^{2}}{\omega \mu \beta d} R_{s}
$$

where $P_{0}$ is the time-average power crossing a transverse section of the waveguide without conductor loss

$$
P_{0}=\frac{1}{2} \iint_{S} \vec{E} \times \vec{H}^{*} \cdot \hat{n} d S=\frac{\omega \mu d W}{4 k_{c}^{2}}\left|B_{m}\right|^{2} \operatorname{Re}\{\beta\}
$$

$P_{l}$ is the power dissipated per unit length in the two lossy conductors

$$
P_{l}=R_{s} \int_{S}\left|\vec{J}_{s}\right|^{2} d S=R_{s} W\left|B_{m}\right|^{2}
$$

and $R_{s}=\sqrt{\omega \mu /(2 \sigma)}$ is the surface resistivity of a PEC. Note in (2.29) that the power flow down the waveguide is zero when $\beta$ is imaginary. This implies that the energy $(\mathrm{E}=h \nu)$ associated with frequencies below this real-imaginary boundary attenuate rapidly within the waveguide, and as such, there is no power transferred by the guide. The frequency associated with this energy is called the cutoff frequency

$$
\nu_{c}=\frac{m}{2 d \sqrt{\mu \epsilon}}
$$

Equation (2.31) shows that the energy transferred by the waveguide is quantized $(m \in \mathbb{N})$. The discrete energies associated with each value of $m$ are called modes, where the lowest energy state supported by the waveguide is commonly called the 
dominant mode. Note that as defined, the domain of $m$ doesn't include 0 . In the TE case, this makes physical sense; if $m=0$, then $E_{x}=H_{y}=0$ and thus $P_{0}=0$.

\section{Transverse Magnetic Modes}

TM modes are defined by $H_{z}=0$. A similar procedure is followed to determine the field components, the conductor loss, and cutoff frequency for the TM case. For brevity, the analysis is not presented. Instead, a summary of the results is as follows:

$$
\begin{aligned}
E_{z} & =A_{m} \sin \left(\frac{m \pi}{d} y\right) e^{-j \beta z} \\
E_{y} & =-j \frac{\beta}{k_{c}^{2}} A_{m} \cos \left(\frac{m \pi}{d} y\right) e^{-j \beta z} \\
H_{x} & =j \frac{\omega \epsilon}{k_{c}} A_{m} \cos \left(\frac{m \pi}{d} y\right) e^{-j \beta z} \\
H_{y} & =E_{x}=0 \\
\alpha_{c} & =2 \frac{\omega \epsilon}{\beta d} R_{s}\left|A_{m}\right|^{2}
\end{aligned}
$$

The propagation constant $\beta$ and cutoff frequency $\nu_{c}$ for the TM case are the same as the TE case. Note though, that $E_{z}$ now has a solution for $m=0$, therefore $m \in \mathbb{N}_{0}$. The dominant mode is now $m=0$, thus $E_{z}=H_{z}=0$. This is by definition the TEM mode, since both the electric and magnetic field are orthogonal to the wave's direction of propagation.

\section{Comparison of Parallel Plate Waveguide Field Distributions}

The field distributions of the TE and TM modes for a PPW are compared in Figures 2.2 and 2.3. There are insightful physical conclusions to be drawn from these comparisons. First, the field is uniform along the length of the waveguide. Second, there 
is a $90^{\circ}$ phase difference between the TE and TM mode. This follows from the analysis of the field components, where $\sin \theta=\cos (\theta+\pi / 2)$. Lastly, even mode numbers $\left(m=\{2 n\} \mid n \in \mathbb{N}_{0}\right)$ produce odd symmetric phase distributions and odd mode numbers $\left(m=\{(2 n+1)\} \mid n \in \mathbb{N}_{0}\right)$ produce even symmetric phase distributions.
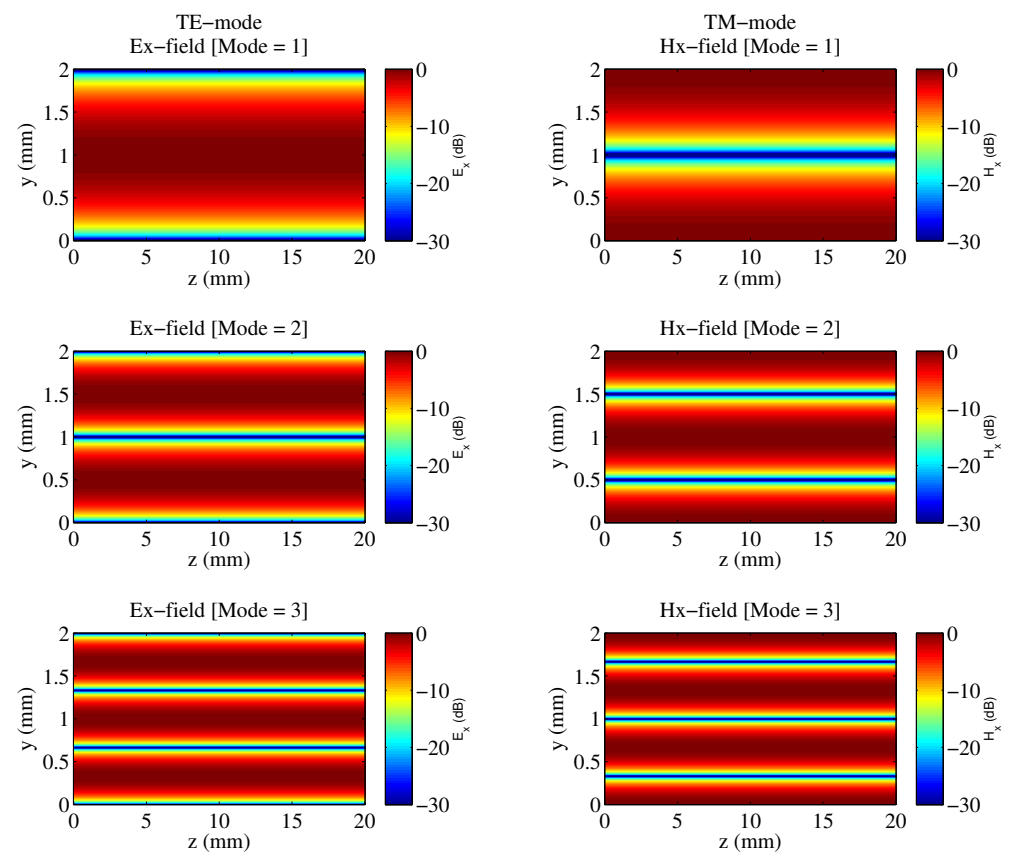

FIgURE 2.2 - Comparison of parallel plate waveguide TE and TM mode field strength distributions.

\subsection{Fourier Transform Mode Matching Technique}

The Fourier transform is widely used in many scientific disciplines. In the field of electrical engineering it is used predominantly to solve signal processing, communication, system control, electromagnetics, and optics problems. The Fourier transform technique is especially well suited for electromagnetics problems since it provides a convenient mathematic representation of wave scattering, diffraction, and 


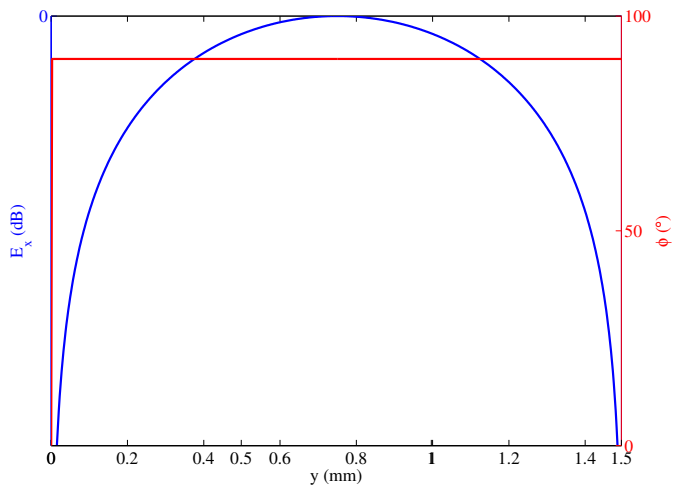

(A) $\mathrm{TE}_{1}$ mode

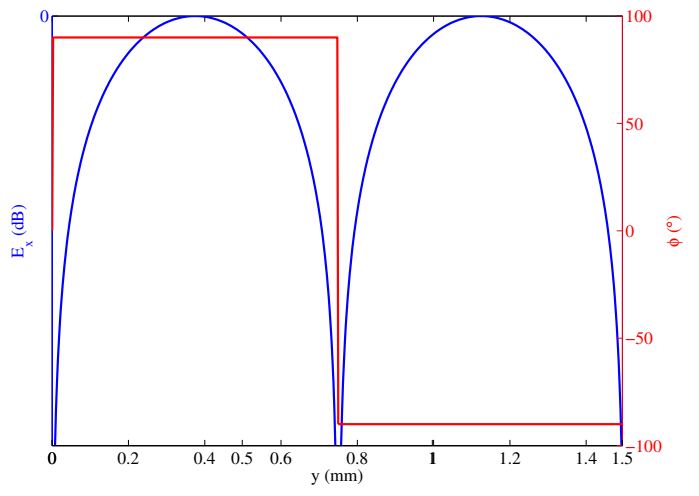

(B) $\mathrm{TE}_{2}$ mode

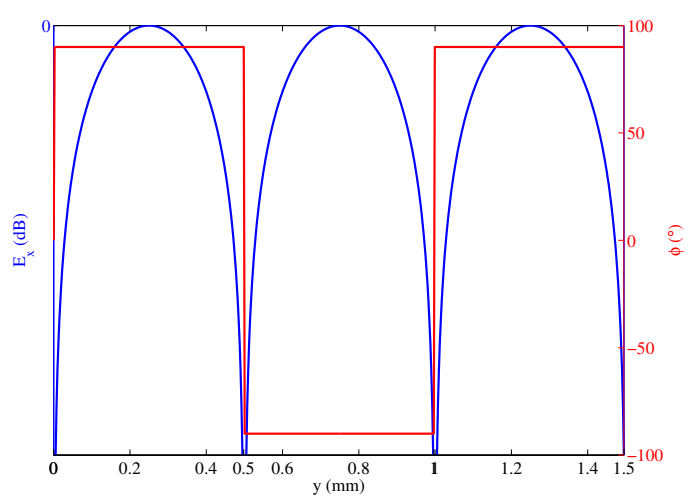

(c) $\mathrm{TE}_{3}$ mode

Figure 2.3 - First three TE field distributions between the plates of the PPW.

propagation. Thus, complex scattering problems often encountered in microwave antenna radiation, diffraction, and interference have been analyzed using the Fourier transform technique. The Fourier transform, mode-matching, and residue calculus offer a robust set of techniques which, when applied to solving EM scattering and boundary problems, obtain simple, analytic, and rapidly converging series solutions. Residue calculus is instrumental in converting solutions into series representations that are efficient and well suited for numerical analysis. The analysis method for scattering and boundary condition problems has the following steps [8]. 
1. Divide the scattering domain into closed and open regions.

2. Represent the scattered fields in their respective regions in terms of the Fourier series and transform.

3. Enforce boundary conditions on the field continuities between regions.

4. Apply the mode-matching technique to obtain the simultaneous equations for the Fourier series modal coefficients.

5. Utilize the residue calculus method to represent the scattered field in a fast convergent series.

The following is a discussion on applying the Fourier transform mode matching technique to TE radiation patterns for a flanged PPW. The derivation presented is an amalgam of material presented in references by Eom et. al [7, 8, 4]. Following the steps outlined prior, the closed region is inside the PPW and the open region is outside. The boundary between these regions is at the free space interface of the waveguide. The geometry for this problem is shown in Figure 2.4. Note, that the orientation of the PPW in this formalization is parallel to the $y$-axis and centered at the origin. This differs from the discussion in Section 2.2, where it is parallel to the $x$-axis and centered at $W / 2$. Repositioning the PPW in this manner changes the field component that designates the TE mode and simplifies the transverse $(x)$ component of the field equations. Next, assuming a time-harmonic variation of $\exp (-j \omega t)$ the fields inside the waveguide are given by

$$
\begin{gathered}
E_{y}^{i}=\sin a_{p}(x+a) \exp \left(j \xi_{p} z\right), \\
E_{y}^{r}=\sum_{m}^{\infty} c_{m} \sin a_{m}(x+a) \exp \left(-j \xi_{m} z\right),
\end{gathered}
$$




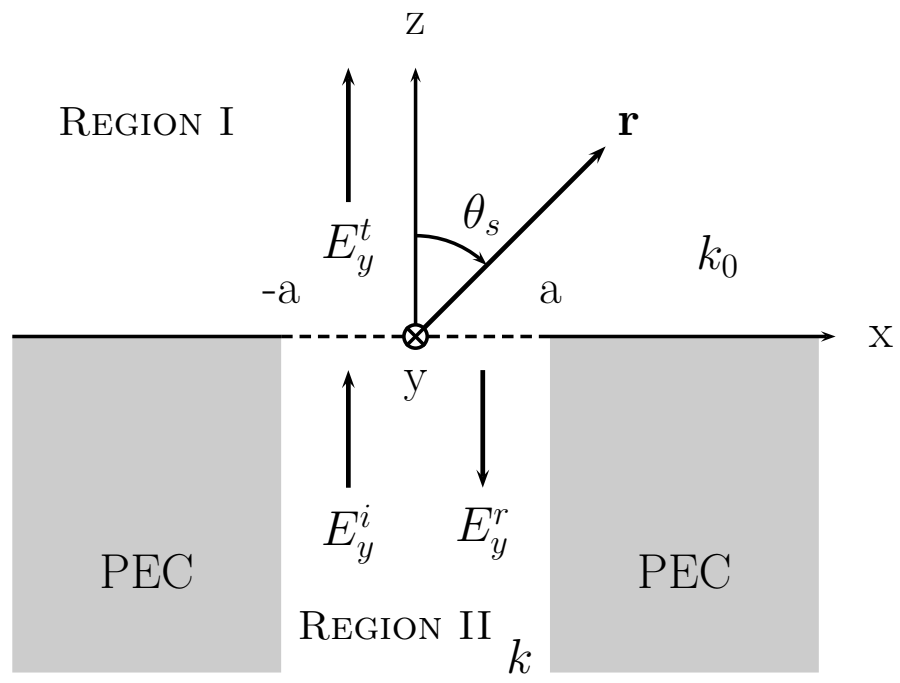

Figure 2.4 - Cross section diagram of a radiation pattern geometry of a flanged PPW.

where $2 a=d$ is the width of the waveguide, $c_{m}$ is the modal reflection coefficient, $\xi_{p, m}=\sqrt{k^{2}-a_{p, m}^{2}}$ is the wavenumber, and $a_{p, m}=p, m \pi / d$ is the eigenvalue. In this case a negative time convention is used for the time-harmonic field representation in order to simplify the integral calculus used for the complex analysis. Note that for odd $p, m=\{(2 n+1)\} \mid n \in \mathbb{N}_{0}$ and for even $p, m=\{2 n\} \mid n \in \mathbb{N}_{0}$. Outside the waveguide the transmitted field represented in the spectral domain $\zeta$ is given by

$$
\begin{aligned}
& E_{y}^{t}(\zeta)=\frac{1}{2 \pi} \int_{-\infty}^{\infty} \tilde{E}_{y}^{t}(\zeta) \exp \left(-j\left(\zeta x-\kappa_{0} z\right)\right) d \zeta \\
& \tilde{E}_{y}^{t}(\zeta)=\int_{-\infty}^{\infty} E_{y}^{t}(x, 0) \exp (j \zeta x) d x
\end{aligned}
$$

where $\kappa_{0}=\sqrt{k_{0}^{2}-\zeta^{2}}$. Next, applying boundary conditions to the tangential components at the interface, the electric field continuity along the $x$-axis at $z=0$ is 
given by

$$
\begin{aligned}
E_{y}^{t}(x, 0) & =E_{y}^{i}(x, 0)+E_{y}^{r}(x, 0) & & |x|<a \\
& =0 & & |x|>a
\end{aligned}
$$

Taking the inverse Fourier transform of $E_{y}^{t}(x, 0)$ yields

$$
\tilde{E}_{y}^{t}(\zeta)=\frac{1}{2 \pi} \sum_{m}^{\infty}\left(c_{m}+\delta_{m n}\right) a_{m} a^{2} F_{m}(\zeta a)
$$

where $\delta$ is the Kronecker delta and

$$
F_{m}(u)=\frac{e^{j u}(-1)^{m}-e^{-j u}}{u^{2}-\left(\frac{m \pi}{2}\right)^{2}}
$$

Continuity of the tangential magnetic field for $|x|<a$ and $z=0$ gives

$$
\begin{aligned}
H_{y}^{t}(x, 0) & =H_{x}^{i}(x, 0)+H_{y}^{r}(x, 0) \\
\tilde{H}_{y}^{t} & =\zeta_{p} \sin a_{p}(x+a)-\sum_{m}^{\infty} c_{m} \zeta_{m} \sin a_{m}(x+a) .
\end{aligned}
$$

Orthogonality of the sinusoidal functions is utilized to obtain simultaneous equations for the modal reflection coefficients $c_{m}$. Equation (2.42) is multiplied by $\sin a_{n}(x+a)$ and both sides are integrated from $a$ to $-a$ wrt $x$ and yields

$$
\begin{gathered}
\frac{1}{2 \pi}\left(I_{p n}+\sum_{m}^{\infty} c_{m} I_{m n}\right)=a\left(\xi_{p} \delta_{n p}-\xi_{n} c_{n}\right), \\
I_{m n}=\int_{-\infty}^{\infty} a^{2} \kappa_{0} F_{m}(\zeta a) F_{n}(-\zeta a) d \zeta .
\end{gathered}
$$


Equation (2.44) is solved by applying contour integration and the calculus of residues. Where the upper half-plane of the contour path is shown in Figure (2.5).

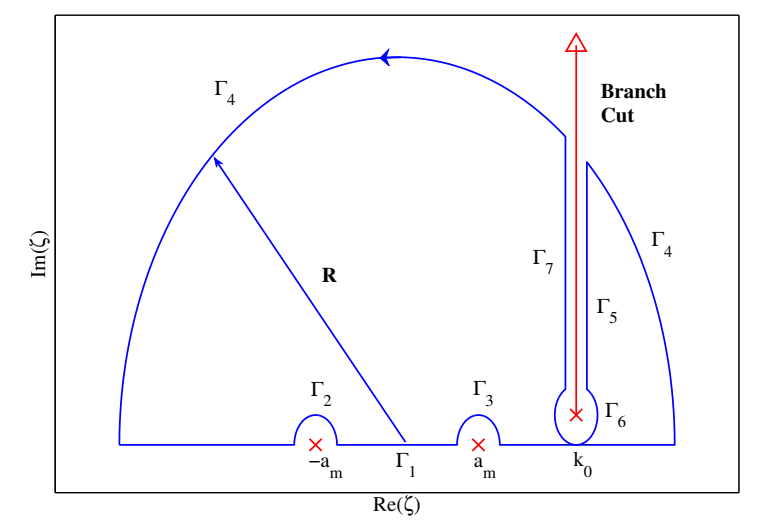

Figure 2.5 - Contour path in the $\zeta$-plane.

Note that when $m+n$ is odd, the integrand is an odd function of $\zeta$, and thus it equals zero. Conversely, when $m+n$ is even, $I_{m n}$ is given by

$$
I_{m n}=\int_{-\infty}^{\infty} 2 \kappa_{0}\left[\frac{1-(-1)^{n} e^{j 2 \zeta a}}{\left(\zeta^{2}-a_{m}^{2}\right)\left(\zeta^{2}-a_{n}^{2}\right)}\right] d \zeta
$$

The integrand has branch points at $\zeta= \pm k_{0}$ and simple poles at $\zeta= \pm a_{m}$ when $m=$ $n$. For analytic convenience, the wavenumber is deformed by adding a infinitesimal positive imaginary part $k_{0}=k_{r}+j k_{i}$. This allows the contour path to remain on the real axis since the branch point has now been moved off it. Applying the residue theorem on the contour $\Gamma$ in the upper half-plane gives

$$
\begin{gathered}
\oint f(\zeta) d \zeta=\oint_{\Gamma} f(\zeta) d \zeta=0, \\
\int_{\Gamma_{1}} f(\zeta) d \zeta=I_{m n},
\end{gathered}
$$




$$
\begin{gathered}
\int_{\Gamma_{2}} f(\zeta) d \zeta=-j \pi \operatorname{Res}_{\zeta=-a_{m}} f(\zeta)=-\frac{\pi \sqrt{\left(k_{0}^{2}-a_{m}^{2}\right)}}{a a_{m}} \delta_{m n} \\
\int_{\Gamma_{3}} f(\zeta) d \zeta=-j \pi \operatorname{Res}_{\zeta=a_{m}} f(\zeta)=-\frac{\pi \sqrt{\left(k_{0}^{2}-a_{m}^{2}\right)}}{a a_{m}} \delta_{m n} \\
\int_{\Gamma_{4}} f(\zeta) d \zeta=0 \\
\int_{\Gamma_{5}} f(\zeta) d \zeta=I_{1 m n} \\
\int_{\Gamma_{6}} f(\zeta) d \zeta=0 \\
\int_{\Gamma_{7}} f(\zeta) d \zeta=I_{2 m n} .
\end{gathered}
$$

Note that $\Gamma_{4}$ contributes nothing to the integral when Jordan's Lemma is applied. Also, $\Gamma_{6}$ also contributes nothing when applying Cauchy's theorem and taking the limit as both the radius and the branch cut width approach zero. Solving (2.47) (2.53) for $I_{m n}$ yields

$$
I_{m n}=\frac{2 \pi}{a} \frac{\sqrt{k_{0}^{2}-a_{m}^{2}}}{a_{m}} \delta_{m n}+\left(I_{2 m n}-I_{1 m n}\right),
$$

where solutions for $I_{1_{m n}}$ and $I_{2 m n}$ are given in [4]. Substituting (2.54) into (2.43) forms the simultaneous equations for the modal reflection coefficients $c_{m}$. When there is no dielectric discontinuity at $z=0, c_{m}$ has the form of

$$
c_{m}=\left(\delta_{m n}-\left[\frac{\left(I_{1 m n}+I_{2 m n}\right)}{2 \pi a\left(\xi_{n}+\sqrt{k_{0}^{2}-a_{m}^{2}}\right)}\right]^{\mathrm{T}}\right)^{-1} \frac{\left(I_{1 p n}+I_{2 p n}\right)}{2 \pi a\left(\xi_{n}+\sqrt{k_{0}^{2}-a_{n}^{2}}\right)} .
$$


The far-field transmitted field [7] based on the saddle point method gives

$$
E_{y}^{t}(r, \theta)=\exp \left[j\left(k r-\frac{\pi}{4}\right)\right] \sqrt{\frac{k}{2 \pi r}} \cos \theta \sum_{m}^{\infty}\left(c_{m}+\delta_{m n}\right) a_{m} a^{2} F_{m}(-k a \sin \theta)
$$

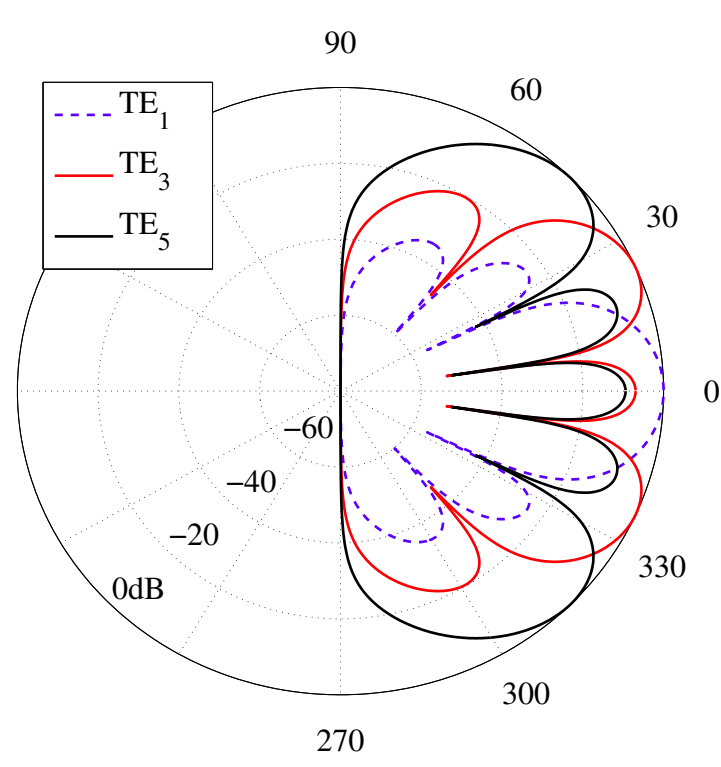

(A) First three even symmetric TE modes.

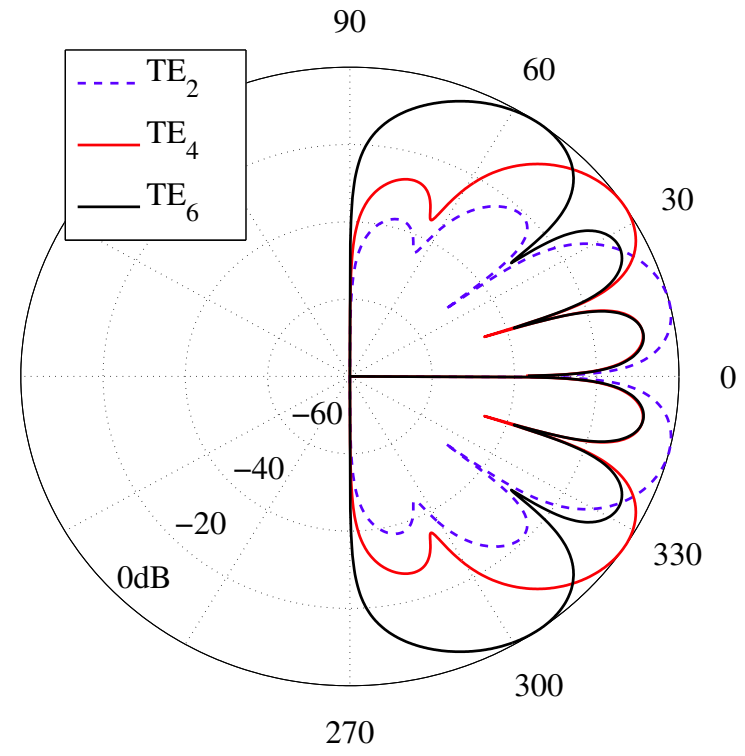

(в) First three odd symmetric TE modes.

Figure 2.6 - Examples of PPW TE radiation patterns using (2.56). (A) indicates the first three even symmetric modes and (B) the odd.

Radiation patterns of the first six TE modes are shown in Figure 2.6. These radiation patterns were created using a plate separation of $0.5 \mathrm{~mm}$ and an incident frequency $\nu$ of $2 \mathrm{THz}$. The first six TE mode cutoff frequencies for this plate separation are shown in Table 2.3. Note that for an incident field frequency of $2 \mathrm{THz}$ the highest order excitable mode is $\mathrm{TE}_{6}=1.8 \mathrm{THz}$. Also, both the lowest order even $(m=1)$ and odd $(m=2)$ symmetric modes have more energy in their main 
lobe than higher order modes. A similar approach for solving the TM radiation pattern for a flanged PPW is presented in [10]. By utilizing a weighted superposition of individual analytic modes, a unique radiation pattern which models the energy coupled into each respective mode can be obtained. This unique radiation pattern corresponds to a unique field distribution at the PPW to free space boundary which identifies which modes are excited. Therefore, by characterizing the radiation pattern of the PPW, mode coupling coefficients can be determined and used on subsequent measurements as a method to compensate for mode conversion loss. This type of procedure is discussed further in Section 5.1.

\begin{tabular}{|c|c|c|}
\hline$m$ & TE $(\mathrm{GHz})$ & TM $(\mathrm{GHz})$ \\
\hline 0 & - & 0 \\
\hline 1 & 300 & $*$ \\
\hline 2 & $*$ & 600 \\
\hline 3 & 900 & $*$ \\
\hline 4 & $*$ & 1200 \\
\hline 5 & 1500 & $*$ \\
\hline 6 & $*$ & 1800 \\
\hline
\end{tabular}

TABLE 2.3 - Flanged PPW TE mode cutoff frequencies for $d=0.5 \mathrm{~mm}$. 
Chapter 3

Finite Difference Time Domain Simulation

\subsection{Mit Electromagnetic Equation Propagation}

MEEP is an open-source implementation of the FDTD method for numerically solving electromagnetic problems. A time domain electromagnetic simulation takes Maxwell's equations and determines the field components as they evolve over time within a finite computational region. An in-depth discussion of the FDTD technique for simulating Maxwell's equations is presented in [23]. The following section gives a general overview of the MEEP software package and how it has been utilized to analyze designs and validate experiments with PPW in the $\mathrm{THz}$ region.

MEEP is free software and is distributed under the GNU's not unix! (GNU) General Public License. It is currently designed for installation on any computer running a Unix based operating system. Instructions for compiling and installing MEEP are available at http://ab-initio.mit.edu/meep. MEEP is also available as precompiled binary packages that have been developed for the Debian and Ubuntu Linux distributions. As part of this work, a port of the MEEP software package was developed for Apple Macintosh. This port is available via the Macports website (http://www . macports.org). If a local installation of the software is not desired, then MEEP is accessible as a resource tool at the NanoHUB website (http://nanohub.org/resources/Meep).

While MEEP is free software, it is by no means less robust or with fewer features than commercially available simulation software. Some of its key capabilities 
are:

- Simulations in 1D, 2D, 3D, and cylindrical coordinates.

- Distributed memory parallelism on any system supporting the message passing interface (MPI) standard.

- perfect matching layer (PML) absorbing boundaries.

- Exploitation of simulation symmetries, which reduces computation size.

- Scriptable front-end or callable from a $\mathrm{C}++$ library or Python interface.

- Output results in the hierarchical data format (HDF5) standard scientific data format.

- Arbitrary material and source distributions.

- Field analyses including flux spectra, energy and power integrals.

The default installation for MEEP installs the $\mathrm{C}++$ interface for use, leaving scripting support and the python interface as optional features. While $\mathrm{C}++$ is a wellestablished programming language, well suited for scientific work, it is recommended that one of the scripting interfaces be configured for use. Doing so offers greater flexibility during development by allowing quick alterations to simulations and simple debugging of script files. Two scripting interfaces to MEEP are currently offered: Libctl, which is written using Guile (GNUs implementation of the Scheme scripting language). The Guile language description is available at http://www .gnu .org/ software/mit-scheme/documentation/mit-scheme-ref/index.html), and Python. While the Scheme scripting language is less well known, it offers access to the full range of $\mathrm{C}++$ library functions and is the interface used here.

To demonstrate the flexibility of the libctl interface a discussion of the general simulation development process is outlined. Employing a top-down design process, the root-level algorithm for a libctl simulation script is as follows: 
1. Define script level parameters - Variables that will change between different iterations of the simulation and are defined as command-line variables that are passed to the simulation script.

2. Define script functions - Typically helper functions or designer output functions but can also include user defined sources or complex media functions as well.

3. Define variables - Global and user-defined variables that are set by the script level parameters or will only be used by a single instance of the simulation.

4. Define simulation cell - The extent of the simulation region in which the fields will be calculated. Typically a simulation cell is surrounded by a PML.

5. Define simulation structure geometries - The objects within the simulation cell, e.g. waveguide geometry and material properties.

6. Define sources - MEEP offers two built-in source function definitions: single frequency continuous-wave and Gaussian pulse. Alternatively, an arbitrary user defined source function could be defined. A source definition includes its geometry and placement in the simulation cell.

7. Define flux regions (optional) - Spatial regions in which MEEP calculates the EM flux normal to the computational volume.

8. Define output functions - Internally these are called step functions. MEEP can output each field component in complex format over a user-defined number of simulation steps. MEEP also offers a wide variety of field calculations, which can be stored in HDF5 binary file format or sent in American Standard Code for Information Interchange (ASCII) format directly to the script output.

9. Post processing - This step typically involves using third party utilities to process the simulation results for analysis or formatting for publishing. 
The outline above offers a general procedure for the development of a MEEP simulation script.

To accurately implement and processes results from a MEEP simulation, it is good to become familiar with the its units (or lack of...) that the software uses for field calculations. MEEP uses scale-invariant units. This means constants like $\mu_{o}, \epsilon_{0}$ , and $c$ all equal unity. This is great for theorists, but becomes cumbersome when real world results are needed for analysis. The following is an overview of working with scale-invariant units.

When performing a simulation, the user must choose a characteristic length $a$ for the system and use that as the unit of distance. For example, consider performing a free space $\mathrm{THz}$ simulation where the frequency range of interest is from 100 Gigahertz $(\mathrm{GHz})$ to $1.2 \mathrm{THz}$. A frequency $\nu$ is selected, approximately in the middle of the band of interest, which will determine the simulation's characteristic length, i.e. $\nu=600 \mathrm{GHz}(\lambda=a=0.5 \mathrm{~mm})$. Converting this to scale-invariant units, $\nu=a / \lambda=1$, and all other quantities should be scaled accordingly. To further illustrate, since $c=1$ in scale-invariant units, $a=a / c$ is the simulation unit of time as well. Suppose that we want to run the $\mathrm{THz}$ simulation and insure that the $1.2 \mathrm{THz}$ components of our source have propagated for 100 time periods ( $\approx 83 \mathrm{~ns}$ ). Since, $\nu=a / \lambda=0.5 / 0.25=2$ and $T=1 / \nu=0.5$, the number of simulation time units to run is $n=T \times 100=50$. Table 3.1 shows some scheme functions that convert international system of units (SI) values into scale-invariant units.

The FDTD method divides space and time into a finite grid (Yee lattice). To obtain accurate simulation results over the region of interest it is beneficial to be familiar with the consequences of this discretization. First, the grid has a spatial resolution $\Delta x$ which gives the number of pixels per unit of distance. This means 


\begin{tabular}{|c|c|c|}
\hline Function & Conversion & Scheme Code \\
\hline$f->m$ & frequency -> scale-invariant & $($ define $(f->m f)(* x f))$ \\
\hline$f<-m$ & scale-invariant -> frequency & $($ define $(f<-m f) \quad(*(/ 1 x) f))$ \\
\hline$t->m$ & time $->$ scale-invariant & $(\operatorname{define}(\mathrm{t}->\mathrm{m} \mathrm{p})(*(/ 1 \mathrm{x}) \mathrm{p}))$ \\
\hline$t<-m$ & scale-invariant $->$ time & $($ define $(t<-m p) \quad(* x p))$ \\
\hline $\mathrm{d}->\mathrm{m}$ & length -> scale-invariant & $($ define $(d->m d)(/ d a))$ \\
\hline$d<-m$ & scale-invariant $->$ length & $($ define $(d<-m d) \quad(* d a))$ \\
\hline \multicolumn{3}{|c|}{$\begin{array}{l}\text { Where a (characteristic length), c (vacuum speed of light), and } \mathrm{x}=\mathrm{a} / \mathrm{c} \\
\text { (scaling coefficient) have all been defined in the script previously. }\end{array}$} \\
\hline
\end{tabular}

TABLE 3.1 - Scheme functions that convert SI values to scale-invariant units.

that the discrete time step $\Delta t$ is given by $\Delta t=S \Delta x$. Where $S$ is the Courant factor and must satisfy condition (3.1) in order for the simulation method to not diverge. In (3.1), $n_{\min }$ is the minimum refractive index of the simulation (typically 1) and \#dim is the number of simulation dimensions (typically 1, 2, or 3). In MEEP $S=0.5$ by default, which is sufficient for most $1 \mathrm{D}-3 \mathrm{D}$ simulations.

$$
S<\frac{n_{\min }}{\sqrt{\# \operatorname{dim}}}
$$

A consequence of this relation is that doubling the spatial resolution halves the temporal resolution. This means that for the same simulation time there are twice as many time steps to compute. As an example, doubling the resolution of a 2D simulation would require $2^{2}=4$ times the memory and would take (at least) $2^{3}=8$ times as much computational time. To insure that the highest frequency of interest is capable of being identified in the grid, an appropriate pixel resolution must be chosen. Second, in order to discretize the EM equations with second order accuracy, 


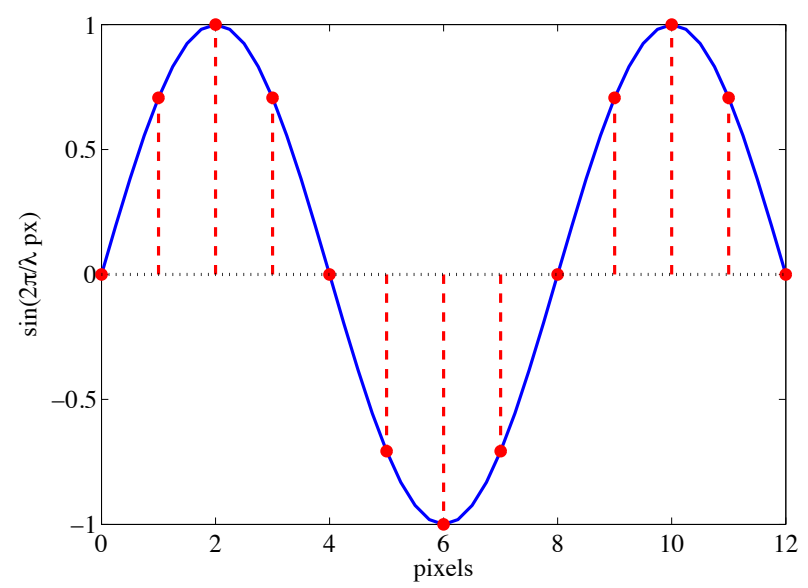

FIgURE 3.1 - Example of FDTD grid spatial resolution required to represent a wave that will achieve second order accurate results.

the FDTD method stores different field components at different grid locations. As a consequence, MEEP interpolates the field components to a common point whenever field calculations or output functions require both electric and magnetic components, i.e. energy density or flux. This interpolation is done internally by MEEP, but is a simple linear interpolation, which means that the field quantities may be less accurate right around dielectric interfaces.

An example of a discretized wave is shown in Figure 3.1, where $\Delta x=$ $10 \mu \mathrm{m} /$ pixel and $a=0.5 \mathrm{~mm}$. In order to collect second order accurate results [5] for a wavelength of $\lambda=250 \mu \mathrm{m}(\nu=1.2 \mathrm{THz})$, the simulation resolution must be set to a minimum of twice that of the simulation's characteristic length. If dielectric materials are introduced into the simulation, the resolution would need to be increased further, due to wavelength shortening $\left(\lambda_{m}=\lambda / \sqrt{\mu_{r} \epsilon_{r}}\right)$ within the material. In general, it is recommended that you maintain at least 8 pixels per wavelength in the highest dielectric material, as illustrated in Figure 3.1.

After a simulation completes, post processing is used to analyze the results. 
MEEP typically stores its results in HDF5 files. The h5utils set of utilities allows for batch visualization and conversion of scientific data in the portable HDF5 format. The h5utils package is free software developed for Unix based operating systems and is available at http://ab-initio.mit.edu/h5utils. Besides providing a simple tool for batch visualization of simulated fields as portable network graphic (PNG) images, it also includes programs to convert HDF5 datasets into universal formats required by other visualization or analysis programs like Vis5d, GNU Octave, and Matlab. Alternatively, HDF5 files can be processed directly in GNU Octave or Matlab. However, h5utils is recommended when handling large data files, i.e. greater than 1 Gigabyte (GB). An isolated time step of the TE field distribution inside a PPW resonant structure is shown in Figure 3.2 to illustrate the insight gained by observing the field distribution. At resonance, the depth of the notches, their pitch, and the center spacing are all approximately $\lambda / 2$ of the incoming wave. For sensing applications of this structure, the sample material should be placed in the area of the highest field concentration, located between the notches in the center spacing [24]. From Figure 3.2, it is apparent that in overmoded operation, the PPW doesn't exhibit this behavior.

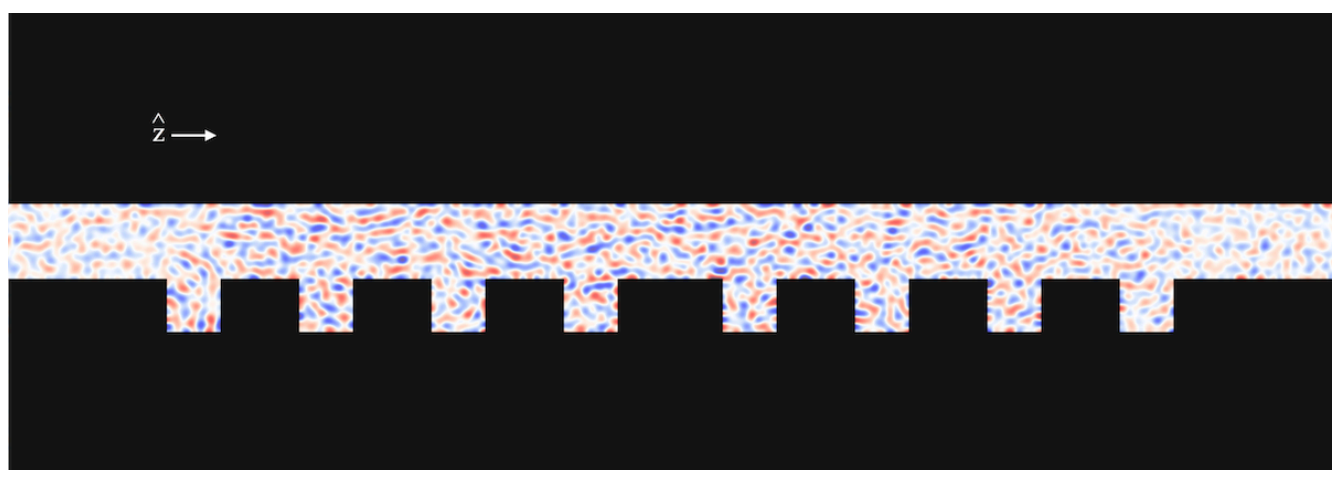

FIgURE 3.2 - Multimode PPW resonator field distribution image created using h5utils (white regions are zero). 


\subsection{Gaussian Source Model}

This section discusses modeling a Gaussian pulse with the same spectral content as the TDS system waveforms for use in MEEP simulations. MEEP has two built-in source functions, continuous-wave and gaussian-pulse. The Gaussian is a useful representation of optical pulses because of its straightforward mathematical description. A Gaussian function is described by

$$
f(t)=\exp \left(-j \omega t-\left[\frac{\left(t-t_{0}\right)}{\sqrt{2} \delta t}\right]^{2}\right),
$$

where $\omega$ is the modulation (source) frequency, $t_{0}$ is the peak time, and $\delta t$ is the temporal width. Note that the full width half maximum (FWHM) of the Gaussian's peak is related to the temporal width by FWHM $=2 \sqrt{2 \ln 2} \delta t$. The Fourier spectrum of a Gaussian function is itself a Gaussian function, where the bandwidth of its

power spectrum is inversely related to the temporal width. The Gaussian function (3.2) is shown in Figure 3.3.

The antennas used in the TDS system operate under the mechanism of photoconductivity, where the optically generated femtosecond pulses of the laser excite free carriers into the conduction band. The delay between these subsequent pulses suggests carrier relaxation/recombination times. The function depicting this type of behavior would exhibit a distinct positive swing when the device was on and a negative swing while the device was off. Therefore, the time derivative of a Gaussian function (gaussian-pulse) is better suited to model the waveform generated by 

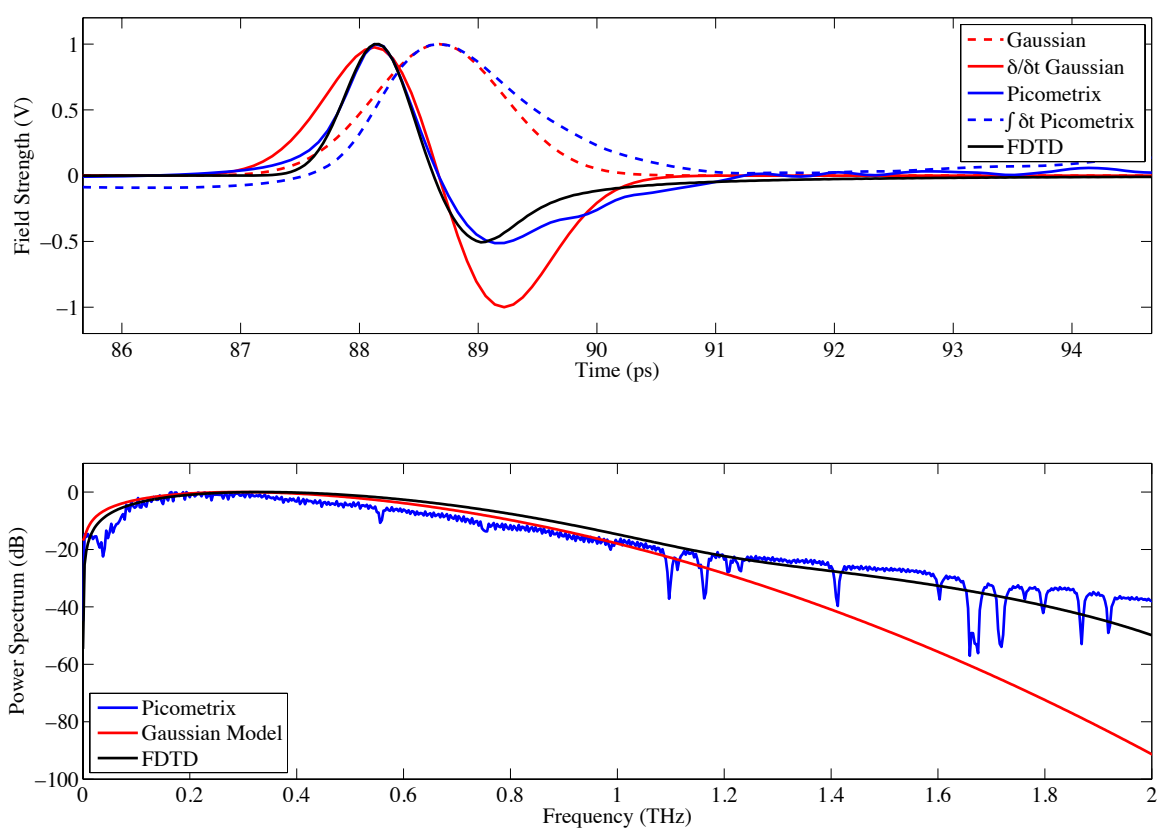

FIGURE 3.3 - Gaussian pulse model of the TDS system waveform compared to the FDTD source function.

a TDS system. The time derivative of (3.2) is

$$
\frac{\partial}{\partial t} f(t)=\exp \left(-j \omega t-\left[\frac{\left(t-t_{0}\right)}{\sqrt{2} \delta t}\right]^{2}\right)\left(1+\frac{\left(t-t_{0}\right)}{j \omega \delta t^{2}}\right)
$$

Note that (3.3) is the summation of two exponentials, the second being proportional to $1 / \delta t^{2}$. This suggests that the difference between (3.2) and (3.3) is negligible. A free space waveform for the TDS system and its corresponding numerical integral and power spectrum are shown in Figure (4.1). Note that the power spectrum was determined by zero padding the 320 ps TDS waveform to 5.120 ns and applying a Gaussian windowing function. Both of these are standard signal processing techniques which allow finer frequency bin resolution and minimize leakage in the side lobes, respectively. From analysis of the time domain (TD) waveform the value for pulse width was determined as $\delta t=545$ fs. From analysis of the power spectrum the 
center frequency was determined as $\omega=60 \pi \mathrm{rad} / \mathrm{s}(30 \mathrm{GHz})$. Note that the TDS system's gaussian-pulse is asymmetric, which increases the half pulse width when the photoconductive (PC) antenna is off. This behavior gives the power spectrum a broader bandwidth. This is due to more high-frequency components required to represent the asymmetric gaussian-pulse in the TD.

The gaussian-pulse implemented in MEEP is given by

$$
\frac{\partial}{\partial t} f(t)=\frac{j}{\omega} \exp \left(-j \omega t-\left[\frac{\left(t-t_{0}\right)}{\sqrt{2} \delta t}\right]^{2}\right) .
$$

A 2D free space FDTD simulation was performed for a gaussian-pulse point source using $\delta t$ and $\omega$ values previously determined by gaussian function analysis of the TDS systems free space waveform. The point source was set at the west end $(-x)$ of the simulation cell and data was collected at the east end $(+x)$ of the cell by integrating the electric field from north to south ( $y$-dimension) of the simulation cell. Agreement is demonstrated (Figure 3.3) between the resulting FDTD simulation and the TDS system waveform and their respective power spectra. 
Chapter 4

MeAsuREMENT Systems

\subsection{Time Domain Spectroscopy}

The generation and detection of broadband $\mathrm{THz}$ radiation in the $\mathrm{TD}$ relies on single-cycle pulses. These pulses are obtained from nonlinear optical responses of electrons in nonlinear crystals or transient photocurrents in semiconductors induced by ultrashort optical pulses from femtosecond lasers. The TDS system used in this work is of the latter variety. The electron responses of this system are not entirely transient, yet are fast enough that the femtosecond optical pulses can induce currents on a sub-picosecond time scale. The specifications of the Picometrix T-Ray ${ }^{\mathrm{TM}} 4000$ TDS system [25] are shown in Table 4.1.

The following is an overview for a typical THz-TDS transmission measurement process. The femtosecond pulse is split into two parts by a beam splitter. One part is translated internally by the system to provide a relative time delay. The other illuminates the emitter and generates the $\mathrm{THz}$ pulse. The $\mathrm{THz}$ pulse travels

\begin{tabular}{|c|c|}
\hline \multicolumn{2}{|c|}{ Picometrix T-Ray } \\
\hline BM & $\mathbf{4 0 0 0}$ \\
\hline Signal-to-Noise Ratio & $>70 \mathrm{~dB}$ \\
\hline Rapid Scan Range & $320 \mathrm{ps}$ \\
\hline Rapid Scan Rate & $100 \mathrm{~Hz}$ \\
\hline
\end{tabular}

TABLE 4.1 - Picometrix T-Ray ${ }^{\mathrm{TM}} 4000$ system specifications. 
through free space, propagates through the material of interest, and then is focused on the detector. The THz-induced transients in the detector are then measured by the relative probe pulses. The $\mathrm{THz}$ pulses are generated and detected by the transient currents in the $\mathrm{PC}$ antennas. The TD waveform and corresponding power spectrum for a TDS free space measurement is shown in Figure 4.1. Note that the secondary pulses in the TD waveform that are located 100 ps from the first pulse. These correspond to reflections from the high-density polyethylene (HDPE) lenses contained in the transmitter and receiver housing. The lenses are approximately $3 \mathrm{~cm}$ from the generating and detecting antennas and are used to focus the $\mathrm{THz}$ beam.

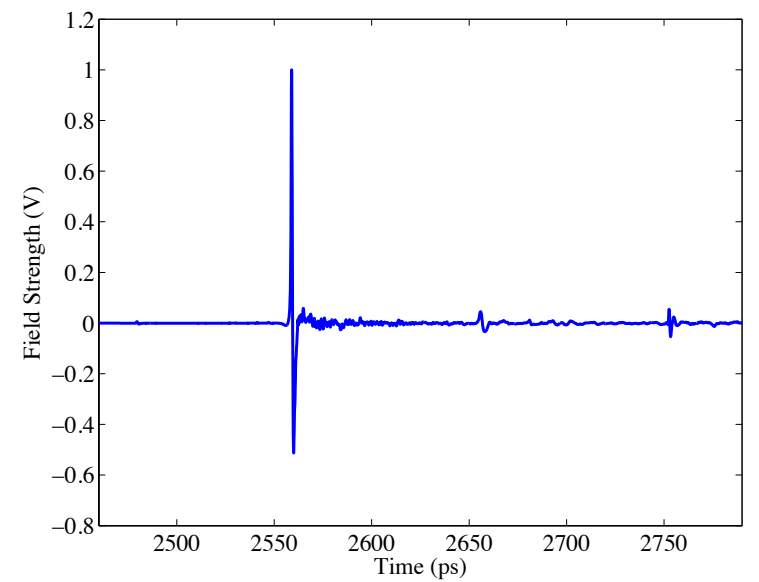

(A) Received waveform

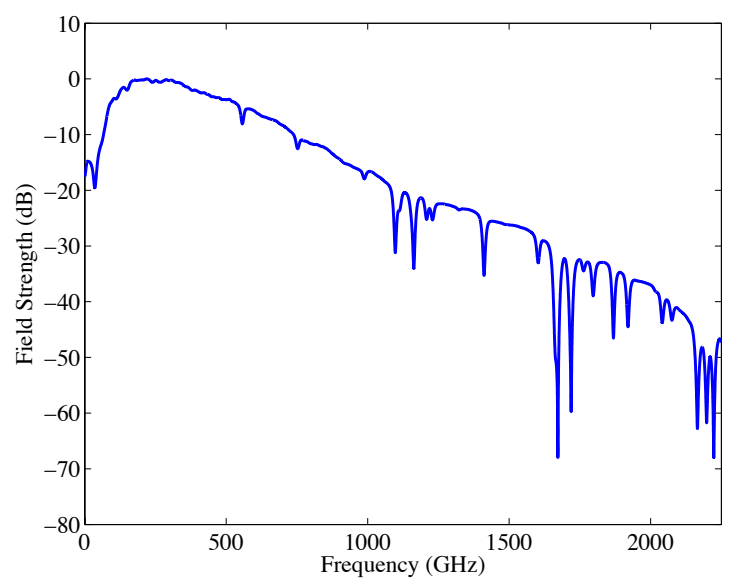

(B) Power spectrum of waveform (A)

FIGURE 4.1 - TDS system free space measurement. (A) Received waveform and (B) corresponding power spectrum.

\subsection{Continuous-Wave Vector Network Analyzer}

The generation and detection of continuous-wave $\mathrm{THz}$ radiation covers a wide range of approaches. In contrast to the broadband $\mathrm{THz}$ radiation methods, they rely on integrating optoelectronic devices such as photomixers and frequency difference 
generators. Examples of solid-state continuous wave (CW) THz emitters are diodebased frequency multipliers, p-type Ge lasers, and quantum cascade lasers. Solidstate sensors used for $\mathrm{THz}$ detection include intersubband detectors and Schottky diodes. Other commonly used $\mathrm{THz}$ sensors are thermal detectors such as bolometers and Golay cells. The CW-VNA system used for this work employs Schottky diodebased mixers and multipliers to emit and sense CW $\mathrm{THz}$ radiation.

\begin{tabular}{|c|c|}
\hline \multicolumn{2}{|c|}{ VDI Extender Modules } \\
\hline Six Waveguide Bands & $75-750 \mathrm{GHz}$ \\
\hline Dynamic Range & $80-120 \mathrm{~dB}$ \\
\hline Compatible VNAs & Aglient, Rhode \& Schwartz, and Anritsu \\
\hline Output Power & up to $5 \mathrm{dBm}$ \\
\hline
\end{tabular}

TABLE 4.2 - Virginia Diodes Inc. (VDI) extender module specifications.

An overview of the CW-VNA systems process for generation and detection of $\mathrm{THz}$ radiation is presented. The CW-VNA system is based on microwave technologies. The system specifications [26] are shown in Table 4.2. The CW-VNA system used for this work was provided by Virginia Diodes Inc. and consists of a set of Vector Network Analyzer (VNA) extender modules configurable in six bands, ranging from 75 - $750 \mathrm{GHz}$. The band designations and their respective dynamic range versus frequency is shown in Figure 4.2 [26].

The operating principle of this type of system involves a microwave synthesizer (e.g. VNA) that is used to seed the solid-state $\mathrm{THz}$ sources. The microwave frequency of the incoming signal is then multiplied using Schottky barrier diodes. Schottky barrier diodes are also employed as microwave detectors. This type of detector typically includes an asymmetric Schottky diode assembly that down converts 


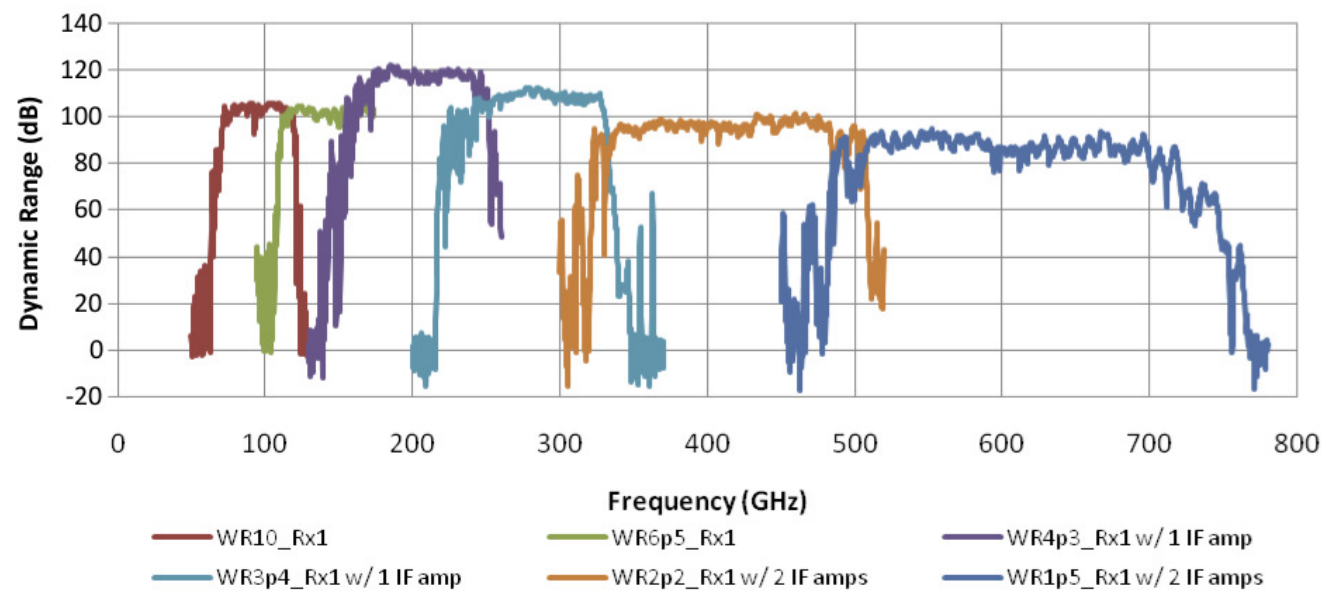

Figure 4.2 - Continuous wave Vector Network Analyzer system dynamic range for WR10 WR1.5 bands.

the incoming signal frequency by nonlinear mixing. To illustrate the operation of high frequency CW devices a brief discussion of the mechanisms in which they are based is presented.

Typical solid-state microwave sources include tunneling (Esaki) and Gunn diodes. Like conventional diodes they are two terminal devices operated in the forward biased region. Unlike conventional diodes, these devices exhibit a distinct negative differential resistance region. When they are tuned to an external resonance, oscillations occur with negligible attenuation and thus radiate a continuous EM wave. In the case of the tunneling diode, within the negative differential region the electrons quantum mechanically tunnel through the potential barrier of the p-n junction. This is essentially the Zener effect, though no reverse bias is needed to initiate the process. The Gunn diode operates on the transferred-electron mechanism, where the semiconductor conduction electrons are shifted from a high-mobility state to a low-mobility state under the influence of a strong electric field. Other solidstate sources include impact avalanche transit-time (IMPATT) diodes which are 
based on negative conductance operated by a combination of carrier injection and transit-time effects.

For the multiplication of microwave frequencies Schottky diodes function as nonlinear media, converting the incoming signal into their harmonic waves. This is analogous to the harmonic generation of optical waves in a nonlinear crystal. The Schottky diode operates using a metal-semicondutor junction known as a Schottky barrier. High conductivity of the metal contact and no semiconductor reverse recovery time results in fast switching. The basic operating principle of these devices is the multiplication of the signal using a nonlinear circuit. The resulting output of such a device are the harmonics of the input signal, which can be described by the Fourier series. The harmonic of interest is then isolated by bandpass filtering.

Schottky diodes are also used as detectors in a frequency mixing configuration. The basic operating principle of such frequency mixers is the beat frequency of two sinusoidal waves.

$$
\sin \omega_{1} t \times \sin \omega_{2} t=\frac{1}{2}\left(\cos \left(\omega_{1}-\omega_{2}\right) t-\cos \left(\omega_{1}+\omega_{2}\right) t\right)
$$

Equation (4.1) shows two sinusoids oscillating at frequencies $\omega_{1}$ and $\omega_{2}$, respectively. These signals are mixed to produce two new sinusoids oscillating at frequencies which are equal to the difference and sum of the original. The difference (beat) frequency is used for down mixing and the summation frequency is used for up mixing. The complete mixing device consists of receiving and emitting antennas for the microwave and $\mathrm{THz}$ signals, input and output waveguides for signal transmission, and a circuit for harmonic-generation. 
CHAPTER 5

Results And Discussion

\subsection{Detection and Estimation of Parallel Plate Waveguide Multi- MOde Operation From Radiation PATterns}

The PPW remains a subject of particular interest in the field of $\mathrm{THz}$ sensing due to the ease in which it can be manufactured, its well defined theory, and multiple modes of operation. Recently, a comprehensive study of the $\mathrm{TE}_{1}$ mode of propagation suggests that it may be best suited for sensing applications [1]. Relatively little work has been done in characterizing PPW operating in a multimode configuration.

When broadband systems are utilized, dominant mode operation is not always achieved because it is possible to couple energy into higher order modes. This is shown in Table 5.1a, where a plate separation of $1.4 \mathrm{~mm}$ corresponds to 8 possible even-order symmetric $\left(m=\{(2 n+1)\} \mid n \in \mathbb{N}_{0}\right)$ TE modes excited up to $2 \mathrm{THz}$. The typical design decision for maintaining dominant mode of operation over the desired measurement band is to modify the geometry of the waveguide. In the case of the PPW, this is achieved by decreasing the plate separation, which increases the dominant mode cutoff frequency and widens the mode bandwidth. To ensure that energy only up to $2 \mathrm{THz}$ propagates in the dominant mode, the PPW plate separation would be decreased to $75 \mu \mathrm{m}$, as is shown in Table 5.1b.

Using this methodology requires multiple waveguides with subsequently smaller dimensions to cover the entire $\mathrm{THz}$ region; in fact, this is what the CW-VNA system 


\begin{tabular}{|c|c|c|}
\hline$m$ & TE (GHz) & TM (GHz) \\
\hline 0 & - & 0 \\
\hline 1 & 107.1 & $*$ \\
\hline 2 & $*$ & 214.1 \\
\hline 3 & 321.2 & $*$ \\
\hline 4 & $*$ & 428.3 \\
\hline 5 & 535.3 & $*$ \\
\hline
\end{tabular}

(A) $1.4 \mathrm{~mm}$ plate separation

\begin{tabular}{|c|c|c|}
\hline$m$ & $\mathbf{T E}(\mathbf{G H z})$ & $\mathbf{T M}(\mathbf{G H z})$ \\
\hline 0 & - & 0 \\
\hline 1 & 2000 & $*$ \\
\hline 2 & $*$ & 4000 \\
\hline 3 & 6000 & $*$ \\
\hline 4 & $*$ & 8000 \\
\hline 5 & 10000 & $*$ \\
\hline
\end{tabular}

(в) $75 \mu \mathrm{m}$ plate separation

TABLE 5.1 - First five PPW cutoff frequencies calculated for TE and TM modes. (A) indicates mode cutoffs for a plate separation of $1.4 \mathrm{~mm}$ and (B) indicates mode cutoffs for a plate separation of $75 \mu \mathrm{m}$.

implements. However, reducing the dimensions of the waveguide introduces propagation loss, group velocity dispersion, and coupling issues [2]. Unlike the CW-VNA system where a selectable frequency range is available, the TDS systems spectral content is contained in its TD waveform. More specifically, the FWHM of its Gaussian pulse width. This gives rise to the PPW operating in a regime in which multiple modes propagate and, as stated previously, leads to excess energy loss in dispersion and mode conversion. Mode-matching techniques [3] have been developed to optimize the PPW input coupling to selectively excite only the dominant mode, but again, these techniques rely heavily on spatial scaling not only of the incident beam but also the geometry of the PPW. By characterizing the behavior of the PPW operating in a multimode configuration corrections may be applied to subsequent measurements which would relax the source and waveguide dimension restrictions that single mode techniques require.

This work investigates the flanged PPW operating in a TE multimode configuration. Measurements presented are taken with broadband TDS and narrowband 
CW-VNA systems. These measurements are compared to analytic solutions using a Fourier transform mode-matching technique [4] and computer simulation using a FDTD technique [5]. Findings from the mode-matching technique comparisons show that multimode operation is identifiable from radiation pattern measurements through a linear superposition of weighted modes.

The flanged PPW was selected for this investigation since multiple numeric approaches and an analytic series solution exist. The analytic series solution used reports the calculated reflection coefficient over a range of waveguide opening to wavelength ratios. It is important to note that the analytic series solution is input independent. Therefore, the analytic series solution may be used to characterize multimode behavior regardless of input coupling, as long as the output is flanged. A boundary-element method has been reported [6] for the case of an arbitrarily flanged PPW. Their findings suggest that other geometries are better suited for directionality and side lobe suppression. Unfortunately, the method reported in [6] does not lend itself to multimode characterization, but a Fourier transform analytic series solution derived from first principles for the geometry in question does.

\subsubsection{EXPERIMENT SETUP}

Measurements were performed on both time and frequency domain systems for cross validation. The TDS system allows for broadband measurements while the CW-VNA system is an excellent validation tool due to its calibration capabilities and higher dynamic range. Figure 5.1 illustrates the experimental setup for characterizing the PPW radiation pattern for both systems. Focusing lenses were used on the transmitter and receiver of the TDS system while horn antennas were used with the CW-VNA. To account for systematic errors, e.g. cabling and connector 


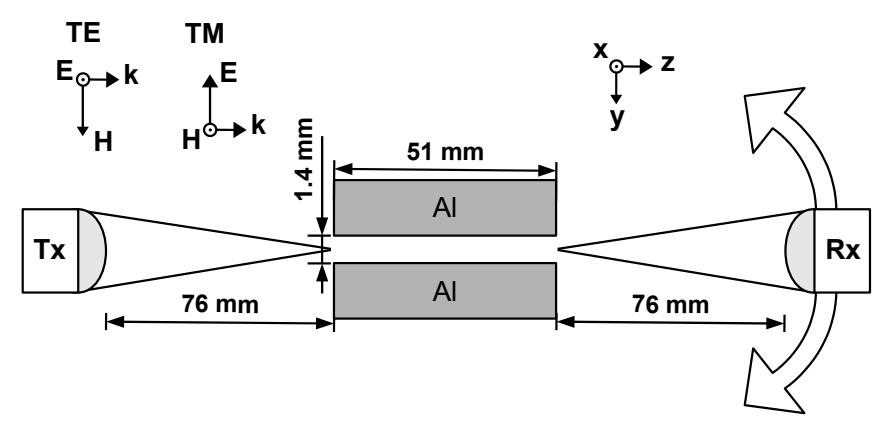

FIGURE 5.1 - Diagram indicating the PPW geometry and setup for a radiation pattern experiment.

losses, the CW-VNA was calibrated to the output of the transmitting and receiving waveguides using a through open short match (TOSM) calibration scheme [27, 28] prior to collecting data for a set of measurements. To account for drift errors in the system: all measurements were taken in a room temperature $\left(20^{\circ} \mathrm{C}\right)$ controlled laboratory, the CW-VNA was allowed the manufacturer's recommended warm-up period [28] (90 min.) prior to calibration, a set of measurements was performed using the same calibration data, and experiments were completed within an 8 hour period.

The top and bottom plates of the PPW were machined out of $50.8 \times 50.8 \times 25.4$ $\mathrm{mm}(l \times w \times h)$ aluminum blocks. Screw threads were tapped in all four corners, which were used to secure the top block to the bottom. This prevented mismatches in alignment of the top and bottom blocks and helps minimize positioning errors between reference measurements. Thin metal washers were used to maintain plate separation when the top and bottom plates were screwed together.

Figure 5.2 illustrates the radiation pattern characterization experiment setup for the TDS and CW-VNA systems. The receiver was mounted on an azimuthal rotation stage in the $y z$-plane and subsequent transmission measurements were performed at $\Delta \phi=2^{\circ}$ increments from $\mp 88^{\circ}$. Data collected on the TDS system 


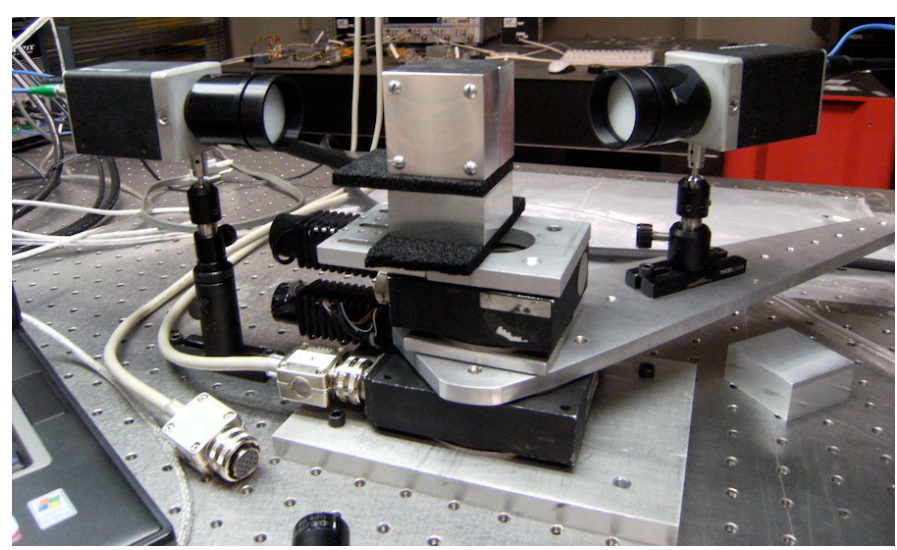

(A) TDS system experiment setup

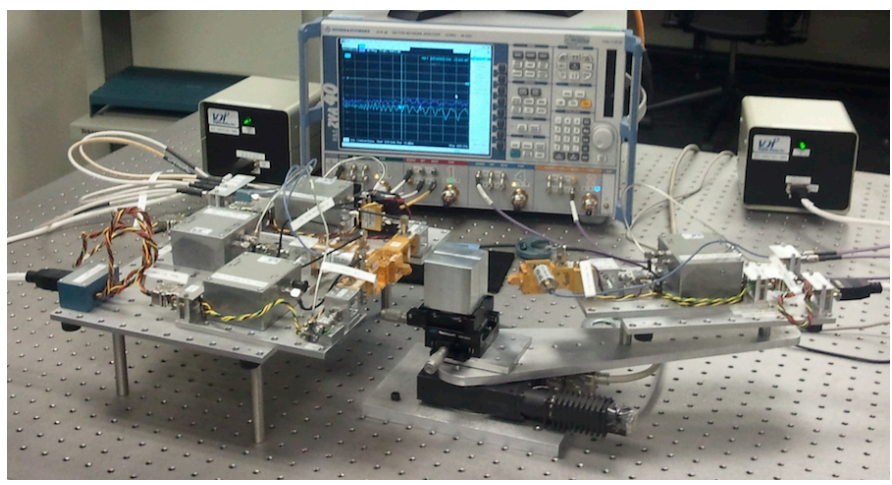

(B) CW-VNA system experiment setup

FIGURE 5.2 - Pictures of the radiation pattern characterization experiments. (A) Shows the TDS system and (B) the CW-VNA system.

was averaged over 6000 waveforms. The CW-VNA system was configured for an intermediate frequency (IF) bandwidth of $10 \mathrm{~Hz}$ and averaged over 10 frequency sweeps. Linearly averaging over waveforms reduces systematic errors in the measurement which increases the system signal to noise ratio (SNR).

CW-VNA and FDTD data presented was collected for a radial distance $r=$ $76 \mathrm{~mm} \approx 3 \mathrm{in}$. This ensured that the observed radiation pattern met the Fraunhofer far-field region criteria for the highest frequency of interest $(2 \mathrm{THz})$, as shown in Table 5.2. This criteria was used because the PPW aperture was larger than a half 
wavelength. In the far-field region, the field components are considered transverse and their angular distribution is independent of the radial distance from the source, i.e. plane waves. The criteria is based on a maximum phase error of $\pi / 8$ radians $\left(22.5^{\circ}\right)$. It has been shown that variations in the amplitude patterns for relative field strengths $\geq 25 \mathrm{~dB}$ are insignificantly affected by such phase errors [29].

\begin{tabular}{|c|c|c|}
\hline Field Region & Criteria & Boundary at 2 $\mathbf{T H z}(\mathbf{m m})$ \\
\hline reactive near-field & $r<0.62 \sqrt{\frac{D^{3}}{\lambda}}$ & 2.7 \\
\hline radiating near-field & $0.62 \sqrt{\frac{D^{3}}{\lambda}} \leq r<2 \frac{D^{2}}{\lambda}$ & $2.7-26$ \\
\hline far-field & $r \geq 2 \frac{D^{2}}{\lambda}$ & 26 \\
\hline
\end{tabular}

TABLE 5.2 - Radiating field regions at 2 THz.

Data collected using the TDS system with focusing lenses was in the radiating near-field (Fresnel) region, as shown in Table 5.2. This criteria was met by positioning the focal point of the lens at a distance $r_{f p} \geq 3 \mathrm{~mm}$ from the PPW aperture. In the near-field the fields phase is dependent on the radial distance from the source. This typically results in a radiation pattern with less defined side lobe nulls. Although, the radiation pattern remains the same as in far-field [29, 19]. Additionally, to remove propagation losses in the field regions, data is presented as normalized radiated power. Table 5.2 summarizes the radiation pattern regions and their respective boundaries at $2 \mathrm{THz}$. Where $D$ is the maximum overall dimension of the aperture and $\lambda$ is the free-space wavelength. Note that for the field region criteria to be valid, $D$ must be larger than the wavelength $\left(D>\frac{\lambda}{2}\right)$. 


\subsubsection{Radiation Pattern Experiment Alignment Process}

The analytic series solution assumes symmetric propagation, therefore, it is critical that the PPW be properly aligned. An example of results for a misaligned PPW operating in the TM mode is shown in Figure 5.3a. Ideally, off axes peaks of the response should occur at the same time regardless of receiver angle. In Figure 5.3a differences of approximately $\pm 3 \mathrm{ps}$ are observed for an angular change of $\Delta 10^{\circ}$. Misalignment of the receiver will result in asymmetric radiation patterns, which leads to inaccurate mode contribution estimates. On the other hand, Figure 5.3b shows a properly aligned PPW operating in the TE mode. After axial rotation by $\pm 30^{\circ}$ the corresponding wave forms are received at the same time.

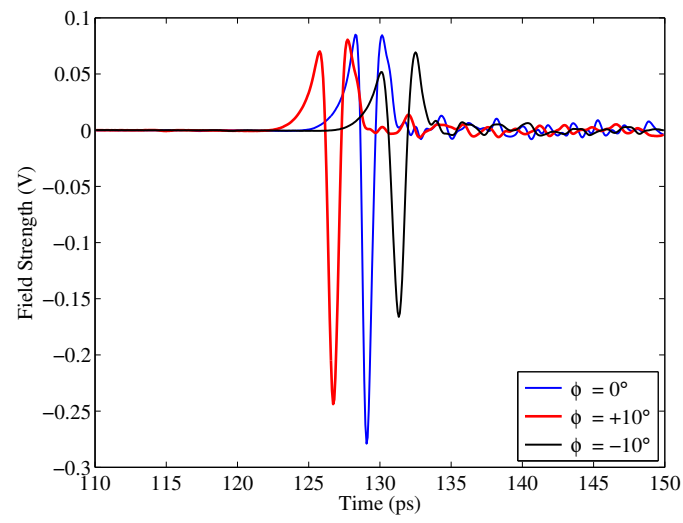

(A) Misaligned TM mode waveforms

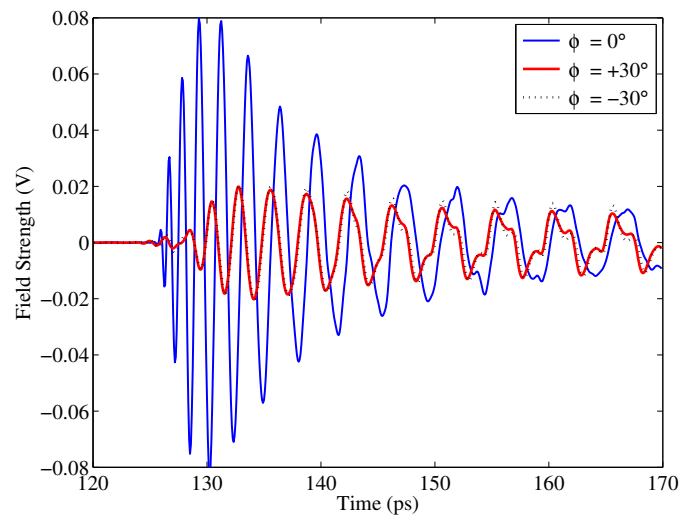

(B) Aligned TE mode waveforms

FiguRE 5.3 - Radiation pattern experiment alignment process time response waveforms. (A) Misaligned TM mode and (B) aligned TE mode.

This demonstrates that the receiver is axially symmetric wrt the center of the flanged PPW. For measurements with a CW-VNA system, axial symmetry is verified by ensuring that the scattering parameter (magnitude and phase) $S_{21}$ at $\pm 15^{\circ}$ and $\pm 30^{\circ}$ are equal. The alignment procedure performed prior to collecting 
data for a radiation pattern experiment is outlined in Figure 5.4.

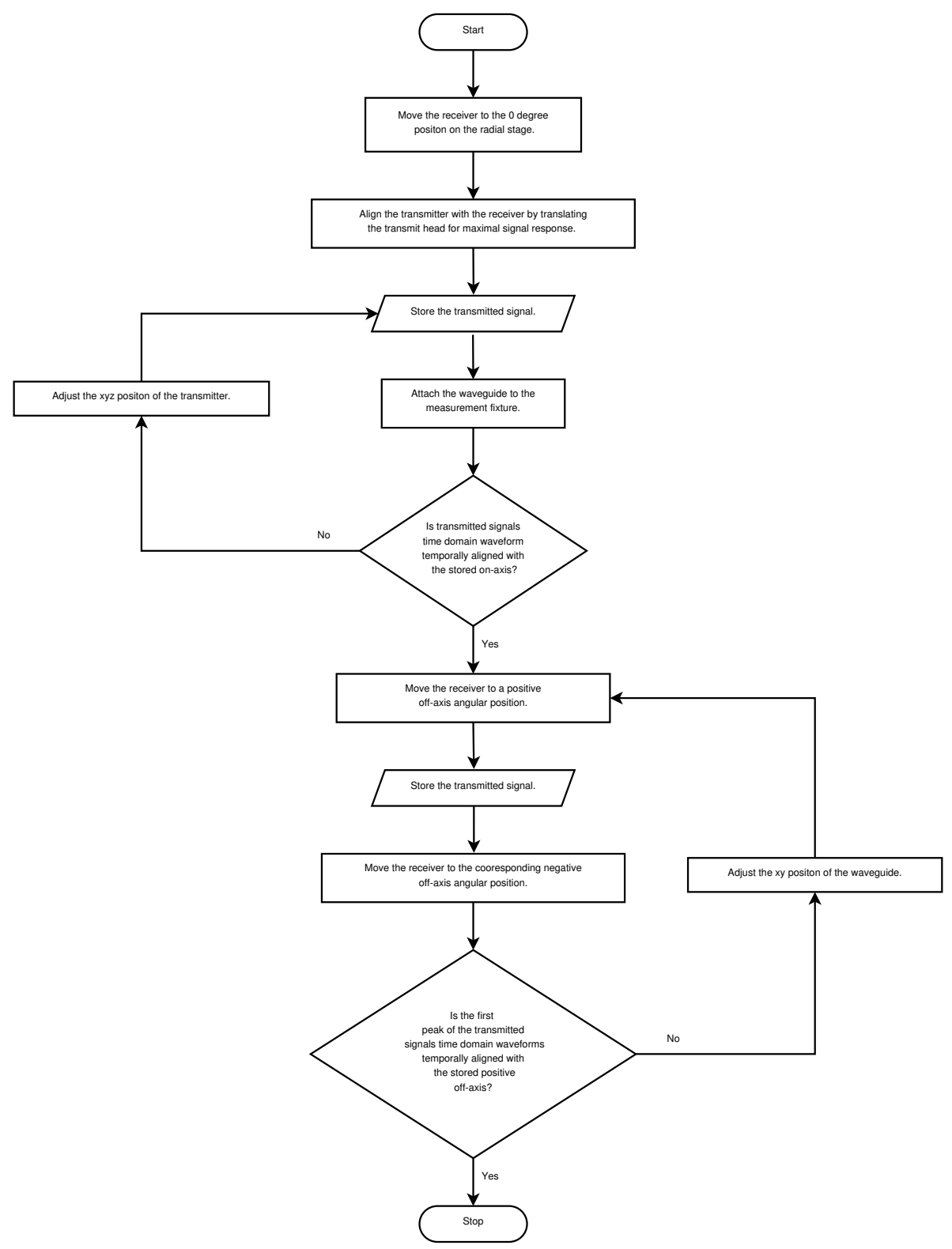

FIGURE 5.4 - Radiation pattern experiment alignment procedure diagram.

\subsubsection{Parallel Plate Waveguide Radiation Patterns}

Measured results from the TDS and CW-VNA systems and FDTD simulation results for TE and TM modes of the field radiated from a flanged PPW are shown in Figure 
5.5 (hi-resolution images displayed on the next page). The onset of the next higher order excited mode is identifiable in the striations of each respective figure. This is especially prevalent at large oblique angles for both the TE and TM mode. While the details of the spectrograms vary from TDS to FDTD to CW-VNA, they all show the same qualitative behavior. In all three cases it is easy to identify the onset of higher order modes at roughly the same frequencies. Note that the CW-VNA data is presented for a much narrower range of frequencies $(330-390 \mathrm{GHz})$ than the TDS and FDTD results $(100-1500 \mathrm{GHz})$. A more detailed examination of these results is the topic of this discussion. 
TDS System Measurements

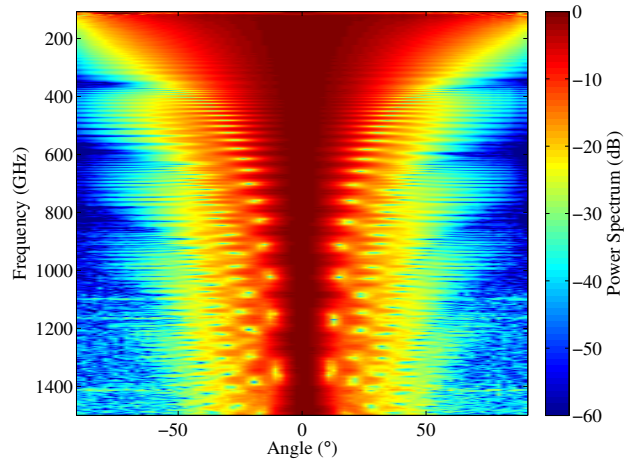

(A) TE mode

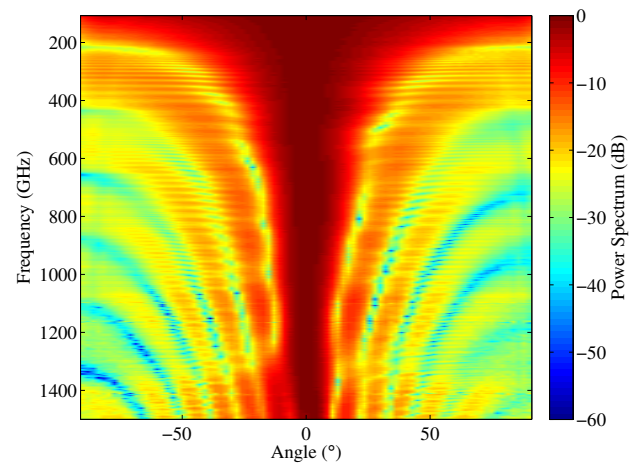

(в) TM mode

FDTD Simulations

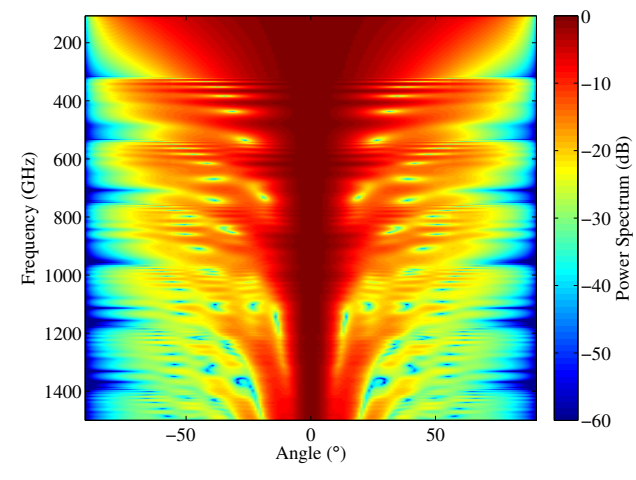

(C) TE mode

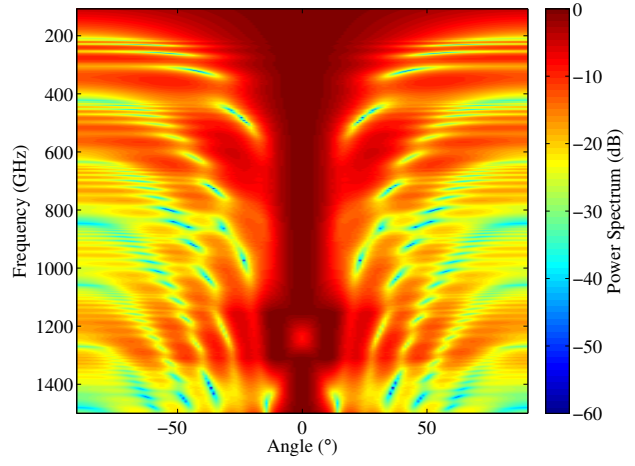

(D) TM mode

CW-VNA System Measurements

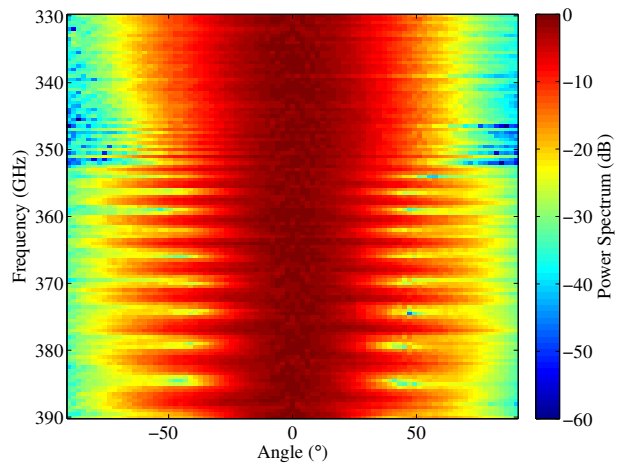

(E) TE mode

FIGURE 5.5 - Measured and simulated radiation pattern spectrograms of the PPW operating in the TE and TM mode. (A) and (B) are TDS system measurements. (C) and (D) are FDTD simulation results. (E) is CW-VNA system measurements. Note that CW-VNA measurements cover the 330 - $390 \mathrm{GHz}$ range, while TDS and FDTD results cover 100 - $1500 \mathrm{GHz}$. 
The Fourier transform mode-matching technique was implemented in a computationally efficient analytic series form [4]. This allowed quick comparisons of measurements to theoretic fields and estimates of the individual mode contributions for any frequency of interest. For example, Figure 5.6 illustrates what the radiation pattern would look like at $0.6 \mathrm{THz}$ if the mode contributions were: $\mathrm{TE}_{1}=80 \%$, $\mathrm{TE}_{3}=15 \%$, and $\mathrm{TE}_{5}=5 \%$.

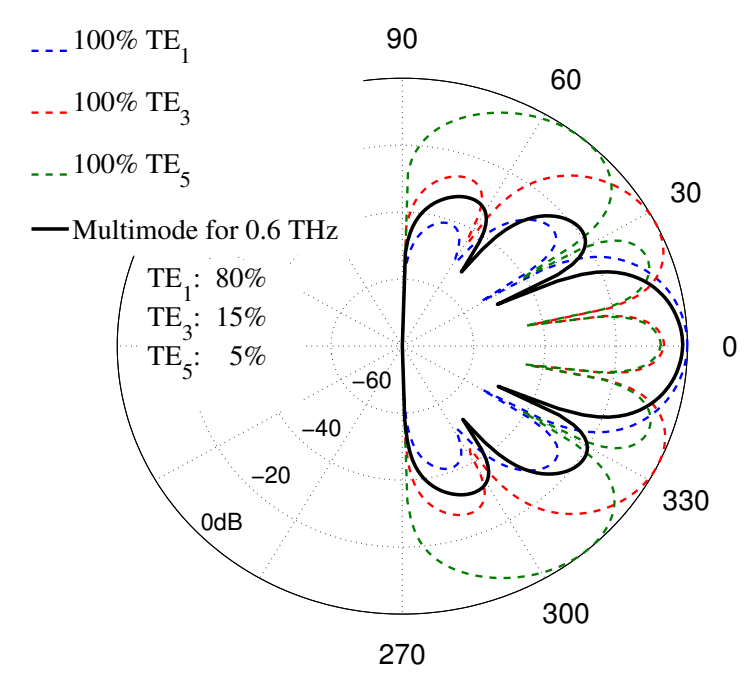

FIgURE 5.6 - Example of the PPW multimode radiation pattern using weighted TE mode contributions of the analytic solution.

To match measured and simulated results with their respective analytic radiation patterns, a BRMSD algorithm was used. This algorithm assumes initially that the dominant mode weighting coefficient is 1 . Then the standard deviation is calculated for each possible radiation pattern weighting, down to 0 . The weighting coefficient with the lowest standard deviation is retained and is subtracted from the remaining total. The process is then repeated using the remaining total as the new assumed mode weighting coefficient for the next higher order mode. A radiation 
pattern composed of the weighting sum of modes is identified when the remaining total is zero or the mode cutoff frequency is higher than the incident frequency.

FDTD simulations were run using the MEEP software suite [5]. These simulations were performed using a broadband TM Gaussian source, as outlined in Section 3.2. Data for the radiation pattern field strength was collected by integrating the field component $E_{y}$ or $H_{y}$ along finite linear segments centered at points that were radial spaced a distance $r$ from the end of the PPW in increments of $1^{\circ}$ from $\pm 89^{\circ}$. Running these simulations offers flexibility in PPW and source geometries for measurement comparison. The FDTD simulations include scattering effects, but unlike the analytic series solution, offer no control over the amount of multimode propagation. Therefore, FDTD simulations offer a more realistic glimpse into how a radiated multimode field should appear. The FDTD simulated radiation pattern for a PPW operating in TE mode is shown in Figure 5.7.

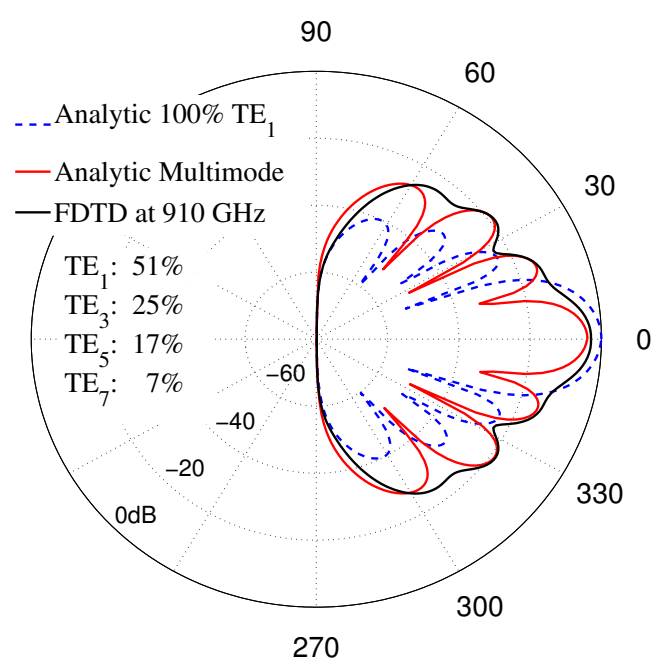

FIGURE 5.7 - PPW TE multimode radiation pattern of FDTD simulation compared to weighted analytic mode contributions. 
Included in this figure is the purely dominant mode radiation pattern and the weighted estimation of higher order modes. With a PPW plate separation of $1.4 \mathrm{~mm}$ the first four even-symmetric TE modes, as indicated in Table 5.1a, are: $\mathrm{TE}_{1}=107$ $\mathrm{GHz}, \mathrm{TE}_{3}=321 \mathrm{GHz}, \mathrm{TE}_{5}=536 \mathrm{GHz}$, and $\mathrm{TE}_{7}=750 \mathrm{GHz}$. Note that inclusion of scattering and interference effects in the FDTD simulation transfers energy into the side lobes and slightly shifts the angular position of the nulls. This effect distorts the distinct side lobes predicted by the analytic solution. Still, deviation from purely first order mode propagation is identifiable.

\subsubsection{Undefined Side Lobe Radiation Patterns}

As shown in Table 5.1a, multimode propagation is expected for an input frequency of $385 \mathrm{GHz}$. The corresponding measurements and the analytic multimode contributions are shown in Figure 5.8.

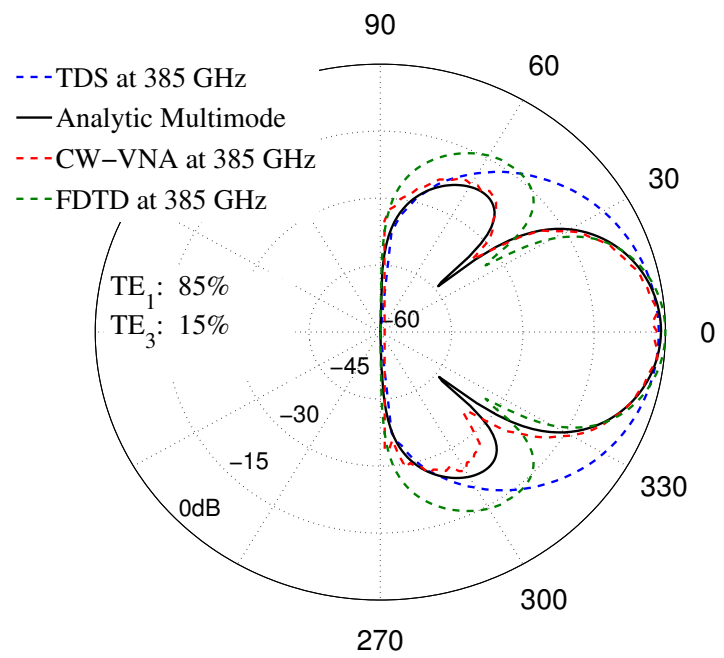

FIgURE 5.8 - PPW TE multimode radiation patterns of TDS and CW-VNA system measurements, and FDTD simulation compared to weighted analytic mode contributions. 
At this frequency the TDS measurement exhibits distortion, where the radiation pattern does not exhibit the side lobes which are characteristic of multimode operation. Therefore, the analytic multimode weighting method cannot be used to predict mode contributions for this experimental result. However, FDTD results show agreement with CW-VNA measurement data, where mode contributions are: $\mathrm{TE}_{1}=85 \%$ and $\mathrm{TE}_{3}=15 \%$. A discussion for this behavior is investigated in the following section.

Higher order mode propagation contributes to reduced forward directionality and greater conductive attenuation loss [3]. The reduced forward directionally is seen in the bounded wave propagation constant $\beta=\sqrt{k^{2}-k_{c}}$, which is a function of frequency and guide geometry. The input frequency is the independent variable for this series of experiments and the PPW geometry is constant, therefore a logical first place to investigate are conductive attenuation loss. As stated previously, the PPW is air filled so dielectric losses do not contribute. In Figure 5.9 the theoretic TE mode conductive attenuation (2.28) were compared to losses extracted from TDS measurements.

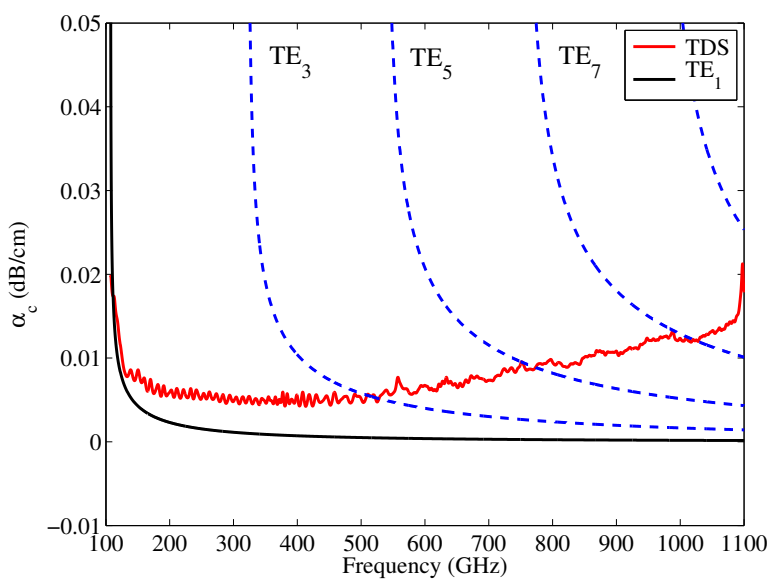

FiguRE 5.9 - Theoretic conductive attenuation loss for even symmetric TE modes compared to TDS system measurements. 
In Figure 5.10a, the amount of excess loss over a small range of frequencies is above their respective theoretic $\mathrm{TE}_{1}$ value. As shown in Figure 5.10b, well defined side lobes appear at the minima in Figure 5.10a, indicated by the black points on the figure. While at the maxima, the corresponding radiation pattern shows no well defined side lobes, indicated by the red point on the figure. This behavior is also observed in the CW-VNA measurements, as indicated in Figure 5.11.

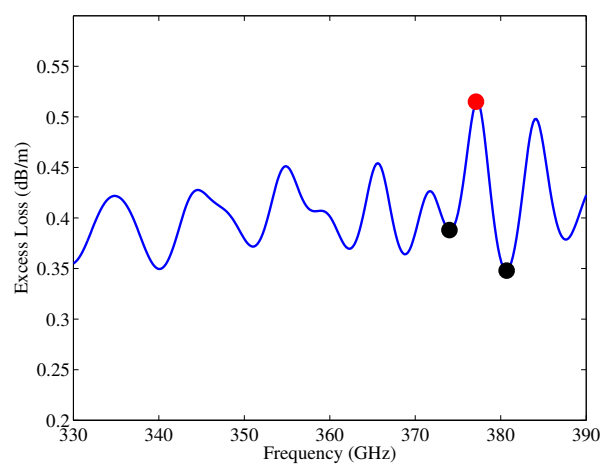

(A) Excess loss

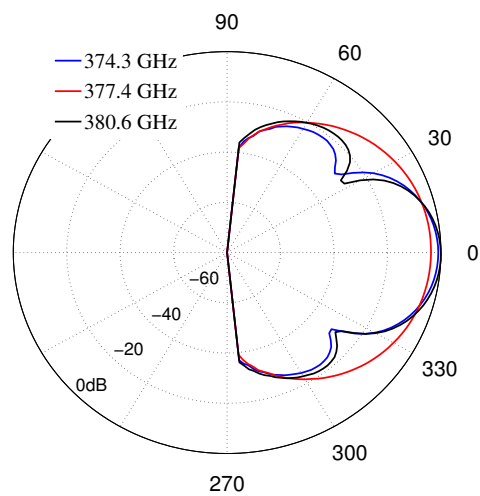

(B) Corresponding TDS system radiation patterns

FiguRE 5.10 - Measured TDS system excess conductive attenuation losses beyond the $\mathrm{TE}_{1}$ mode. Black points indicate radiation patterns with distinct side lobes. Red point indicates a radiation pattern without the expected side lobes. (A) Excess loss in TDS measurements and (B) the corresponding radiation patterns for indicated points. 


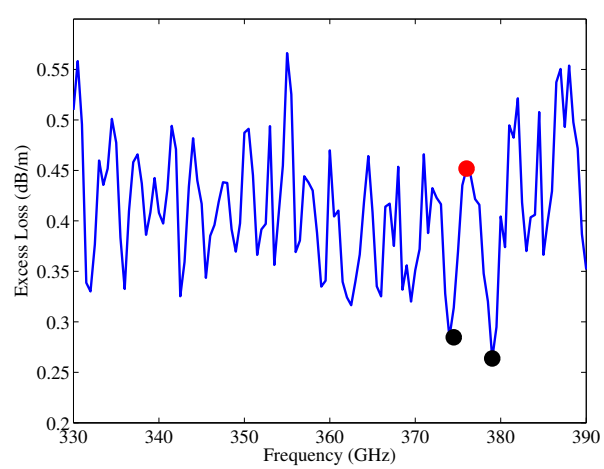

(A) Excess loss

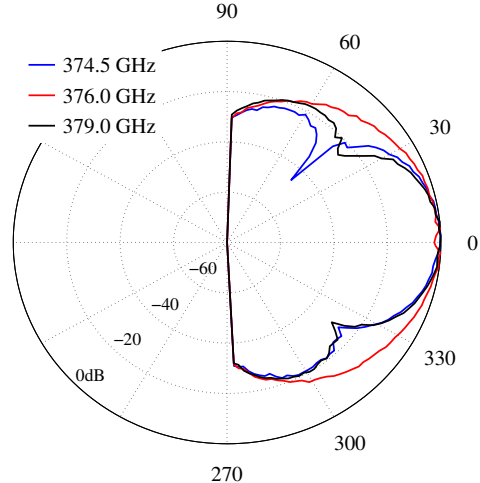

(B) Corresponding CW-VNA system radiation patterns

FiguRE 5.11 - Measured CW-VNA system excess conductive attenuation losses beyond the $\mathrm{TE}_{1}$ mode. Black points indicate radiation patterns with distinct side lobes. Red point indicates a radiation pattern without the expected side lobes. (A) Excess loss in CW-VNA measurements and (B) the corresponding radiation patterns for indicated points.

To illustrate that FDTD simulation also exhibits the same oscillatory behavior between well defined and undefined side lobe radiation patterns it is compared to CW-VNA results in Figures 5.8 and 5.12. A frequency of $383 \mathrm{GHz}$ corresponds to a maxima point similar to those presented on Figures 5.10a and 5.11a. In Figure 5.8, the well defined side lobes in the radiation pattern measurement allow the analytic multimode weighting estimations. This demonstrates that, as in the TDS system measurements, the CW-VNA measurements and the FDTD simulations exhibit the same variations between well defined and undefined side lobe radiation patterns. Further, the exact frequencies in which undefined side lobes occur is inconsistent across the entire band for both systems and simulation. Taken separately, each result can be matched to a radiation pattern with well defined side lobes when the excess loss is at a minimum. Some overlap is observed across measurements and simulation, as shown in Figures 5.8 and 5.12. Despite this inconsistency, the 


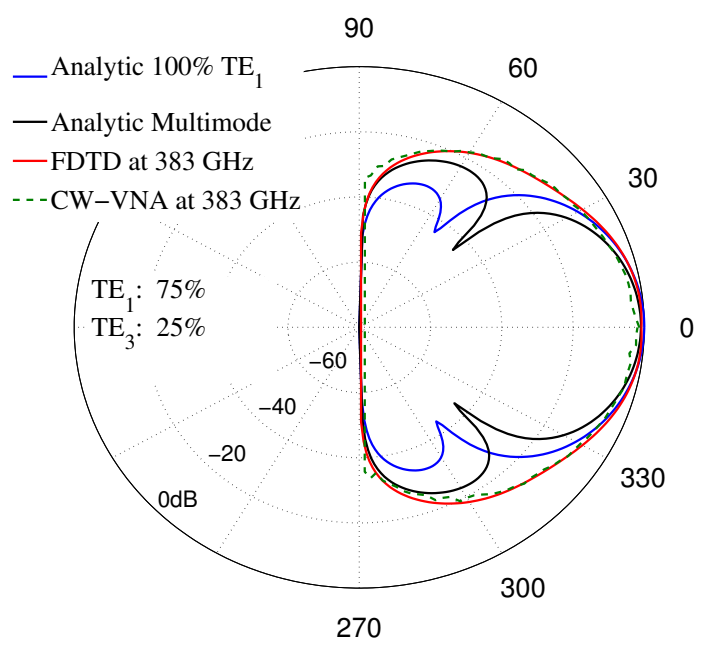

FIGURE 5.12 - TE multimode radiation patterns of FDTD and CW-VNA at $383 \mathrm{GHz}$ compared to weighted analytic mode contributions.

fact that this behavior is observed for all indicates that it is not an artifact of the measurement systems or simulation but a physical phenomenon. The mechanism behind the oscillatory behavior of this excess loss and the distortion that is observed in the corresponding radiation patterns is not well understood at this point and merits further investigation. Although, disagreement across the experiments of the exact frequencies at which undefined side lobes occur is attributed to alignment mismatches in the measurement systems as compared to the exact geometries used for simulation.

In comparing other FDTD simulations with measurements it was observed that all simulations appear to contain a larger percentage of higher order modes than predicted by the analytic solution. To illustrate this Figure 5.13 shows a weighted analytic solution fit to TDS measured results at $910 \mathrm{GHz}$. Comparing these findings with the results in Figure 5.7, demonstrates that in the former case $\mathrm{TE}_{1}$ is estimated 


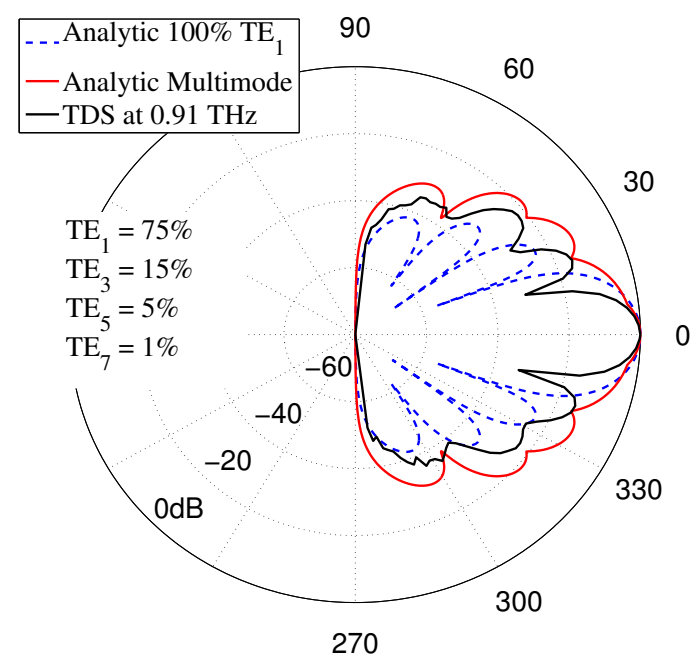

FIGURE 5.13 - TE multimode radiation patterns of FDTD and TDS at $910 \mathrm{GHz}$ compared to weighted analytic mode contributions.

to be $76 \%$ versus $51 \%$. To limit ambiguity in mode weighting estimations, this method should only be applied to frequencies that exhibit well defined side lobe radiation patterns.

As stated in Section 2.3 an analytic solution for the TM radiation pattern is presented by Kim et. al [10]. Measurements were preformed on the TDS system and compared to FDTD simulations in Figure 5.14. Measurements were not performed on the CW-VNA system due to an inability to easily mount the receiver and rotation stage in an orthogonal orientation wrt the PPW opening and maintain the necessary polarization in the $\phi$-plane. As shown in Figure 5.14, multimode operation is also identifiable and mode weighting coefficients can be determined for the TM mode. For the same frequencies of interest, the TM dominant mode weighting coefficients are greater than the TE mode. This behavior is expected since the $\mathrm{TM}_{0}=\mathrm{TEM}$ mode is the first excited mode for an incident plane wave coupled into the PPW. 


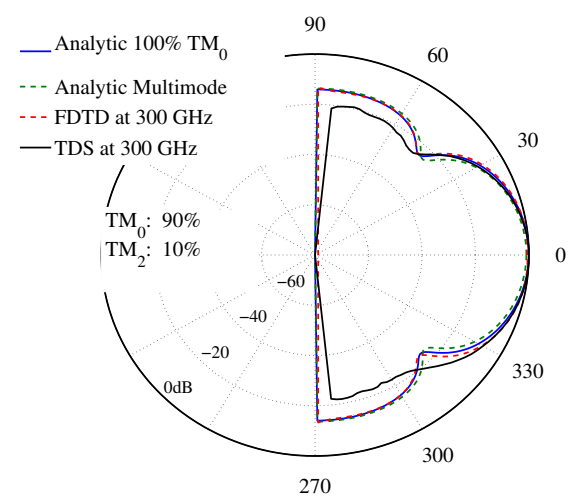

(A) $300 \mathrm{GHz}$

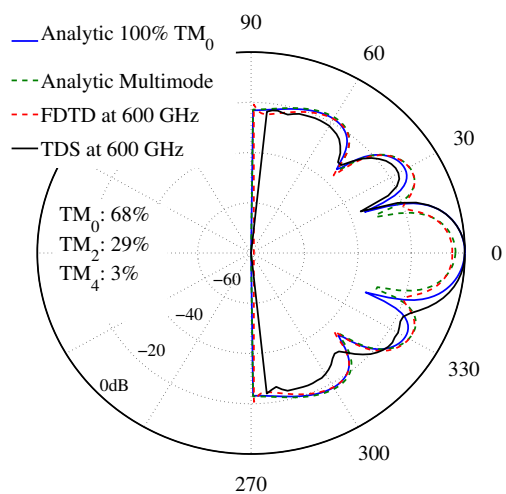

(B) $600 \mathrm{GHz}$

FIgURE 5.14 - TDS radiation pattern measurements of a flanged PPW operating in TM multimode compared to FDTD simulations and theoretic weighted analytic solution. (A) Input frequency of $300 \mathrm{GHz}$ and (B) at $600 \mathrm{GHz}$.

\subsection{Parallel Plate Waveguide Resonant Structures}

\subsubsection{Single Notch}

The efficient detection of biomolecules is increasingly important in the biological, medical, and pharmaceutical industries. Standard techniques often include costly and cumbersome marking processes to identify biomolecules. THz spectroscopy is well suited as an alternative since many biomolecules have spectral signatures in this region of the EM spectrum. Important attributes of a biosensor system are performance and cost efficiency. The integration of system components on chip serves to increase performance and reduce over all system size. However, these systems typically use low temperature semiconductors (i.e. LT-GaAs, InP) with high electron mobilities for photoconductive switches that generate and detect $\mathrm{THz}$ signals. The fabrication processes for these devices are expensive and ill suited for biological applications that require disposable sensors. In contrast, the transmission lines 
(waveguides) and biosensing resonant structures are passive components, which are inexpensive to fabricate. By separating the expensive active components from the cheaper passive components a modular biosensor system with cheap disposable sensors is realized.

In the following section, single notch and periodically notched PPW resonant structures are investigated for use as $\mathrm{THz}$ biosensors. To date, there are no known works that link the characteristic design parameters of these structures to their peak resonance. Field solutions for similar structures has been presented [7, 8]. These solutions lend themselves to computationally efficient close-form expressions for the structure's transmitted power. However, these analytic solutions only cover simple rectilinear geometries. This leaves the typical design process for these devices to iterative EM simulations where design parameters are refined to meet their respective specification. This work focuses on characterizing the effects of two major design parameters: notch depth and plate separation. The effects of modifying the the single notched parallel plate waveguide (N-PPW) notch depth is investigated by FDTD simulations. Then TDS and CW-VNA system measurements are compared to FDTD simulations of the notched periodic parallel plate waveguide (NP-PPW) with varying plate separations.

The geometry of the single notch PPW resonator is shown in Figure 5.15. Where $d$ is the plate separation, $w$ is the notch width, and $t$ is the notch depth. For this set of simulations $w=412 \mu \mathrm{m}, d=1 \mathrm{~mm}$, and $t=\{206,412,824,2060\} \mu \mathrm{m}$. 


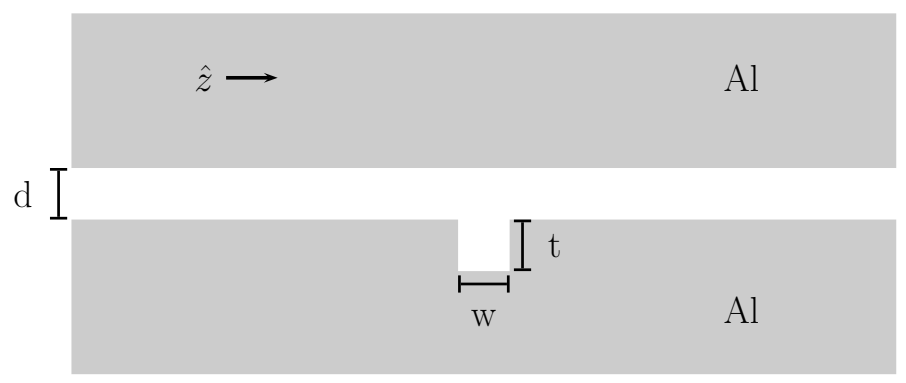

FiguRE 5.15 - Cross section diagram of the N-PPW resonant structure.

FDTD simulations were performed using a broadband TE Gaussian source, as outlined in Section 3.2. Data for the transmitted field strength was collected by integrating the mode component $E_{y}$ across plate separation at the end of the N-PPW. Prior to collecting data for these experiments the FDTD simulation was verified using published results [30].

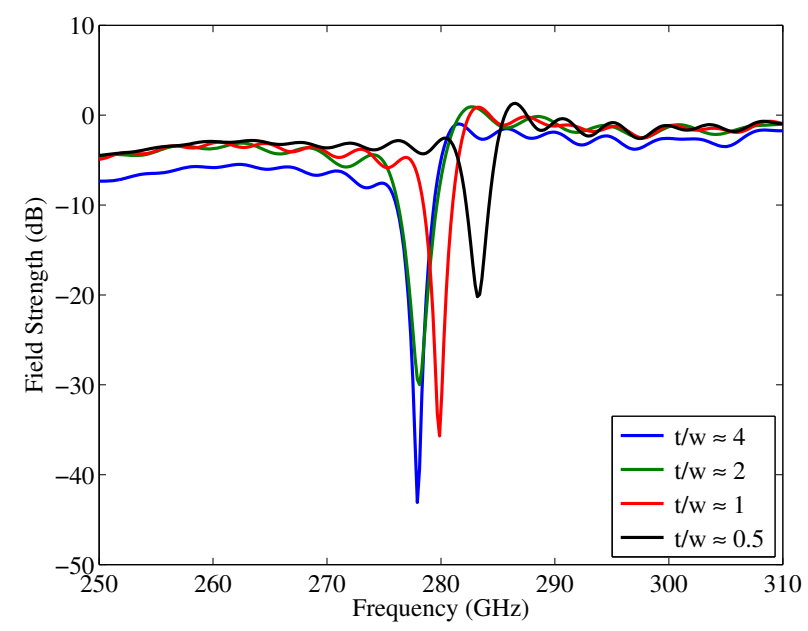

Figure 5.16 - FDTD simulation results illustrating resonant peak shift for varying N-PPW depth-to-width ratios $(t / w)$.

Results from FDTD simulation presented in Figure 5.16 indicate that relative to the case where $t / w=1$ the resonant peak increases for smaller depths and decreases for larger depths. There is a specific depth-to-width ratio which limits the 
resonant peak shift. Table 5.3 summarizes the notch depths and their corresponding resonance and depth-to-width ratio. Results indicate that the resonant peak shows little dependence on the notch depth when the ratio of depth-to-width is greater than 2. For practical reasons the depth-to-width should be limited to less than 2 .

\begin{tabular}{|c|c|c|c|}
\hline Depth $(\mu \mathrm{m})$ & Resonance $(\mathbf{G H z})$ & Ratio & $\Delta \nu(\%)$ \\
\hline 206 & 283 & 0.5 & 1.07 \\
\hline 412 & 280 & 1 & 0 \\
\hline 824 & 278 & 2 & 0.71 \\
\hline 2060 & 277 & 4 & 1.07 \\
\hline
\end{tabular}

TABLE 5.3 - Resonant frequency values of simulated FDTD single notch depths.

\subsubsection{Periodically Notched}

The geometry of the NP-PPW resonator is shown in Figure 5.17, where $d$ is the plate separation, $w$ is the notch width, $t$ is the notch depth, $p$ is the pitch between notches, and $g$ is the center span. For this set of experiments $w=112 \mu \mathrm{m}, t=93 \mu \mathrm{m}$, $p=280 \mu \mathrm{m}, g=162 \mu \mathrm{m}$, and $d=\{100,200\} \mu \mathrm{m}$.

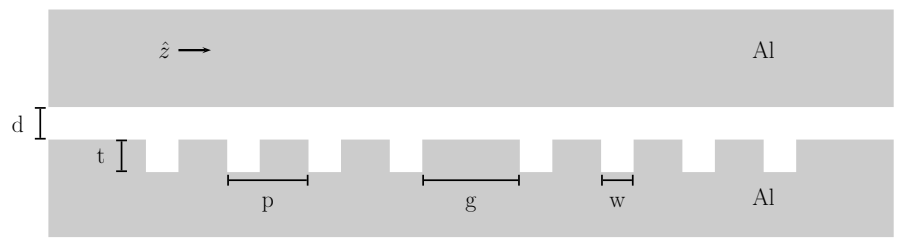

FIgURE 5.17 - Cross section diagram of the NP-PPW resonant structure.

For a plate separation of $d=100 \mu \mathrm{m}$, the dominant mode cutoff frequency $\mathrm{TE}_{1}=1.5 \mathrm{THz}$. This is at the limits of the TDS system SNR and well above the highest CW-VNA system band described in Chapter 4. Therefore, the waveguide is operated in the TM mode, which ensures that there is no low frequency cutoff. 
The top and bottom plates of the NP-PPW were machined out of $50.8 \times$ $50.8 \times 25.4 \mathrm{~mm}(l \times w \times h)$ aluminum blocks with a $6^{\circ}$ taper milled out of each end leaving a $25.4 \mathrm{~mm}$ planar surface in the middle of each block. The $6^{\circ}$ taper at the input and output of the NP-PPW was included to improve free space coupling efficiency [13]. Two inset positioning pins were placed in diagonal corners of the bottom block to be used as guideposts. In addition, screw threads were tapped in the opposite diagonal corners, which were used to secure the top block to the bottom. The crossed guideposts and screws were used to prevent mismatches in alignment of the top and bottom blocks. Screw threads were tapped in the top and side of the blocks in order to secure the entire NP-PPW to the measurement platforms. This fixes the bottom plate of the waveguide so that only the top plate is exchanged between measurements, which helped minimize positioning errors between sample and reference measurements. The central $25.4 \times 25.4 \mathrm{~mm}$ planar area was reserved for machining the resonant structure. Thin metal washers were used to maintain plate separation when the top and bottom plates where screwed together.

The top plate of the waveguide was micro-milled and a machining analysis was performed to verify the dimensions of the channels. A picture of the periodically notched structure is shown in Figure 5.18. The results from the dimensional analysis of the device indicate that channel dimensions deviate from their nominal value by $\sigma<5 \mu \mathrm{m}$. The tapered ends of the NP-PPW visible in Figure 5.18A increase coupling .

Transmission measurements where performed on both TDS and CW-VNA systems for co-validation. Collimating lenses were used for the TDS system experiments and horn antennas were used for the CW-VNA system experiments. For both experiments the transmitter and receiver antennas where aligned for maximal signal 


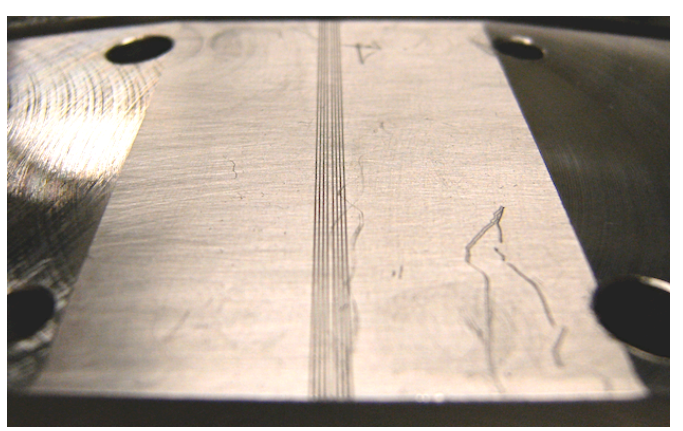

(A) Full resonator image

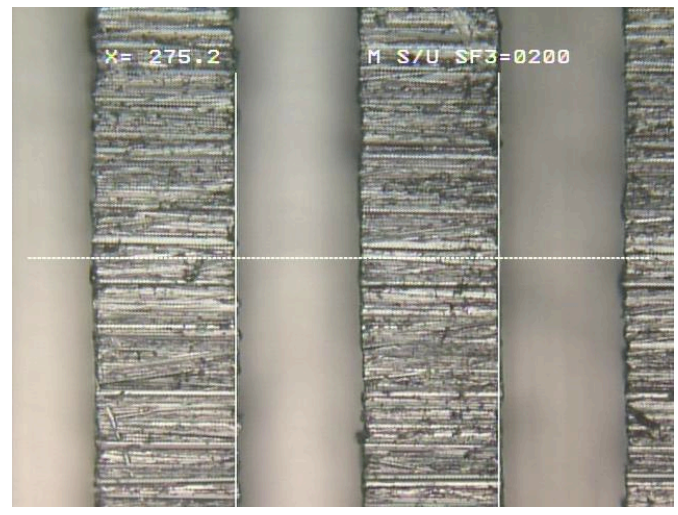

(c) Milled notch pitch

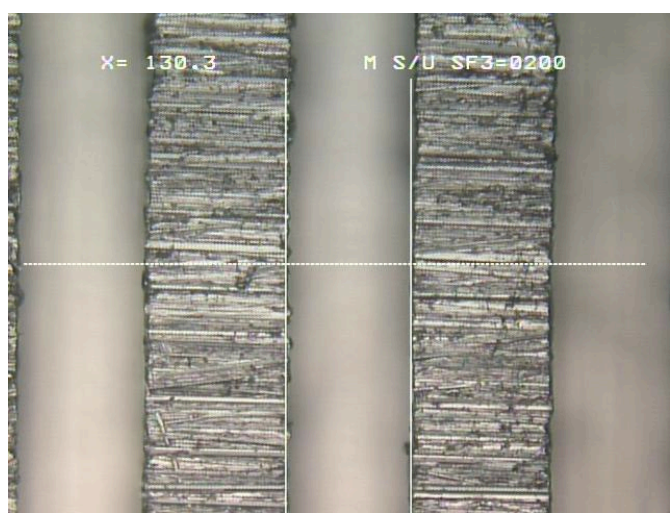

(B) Milled notch width

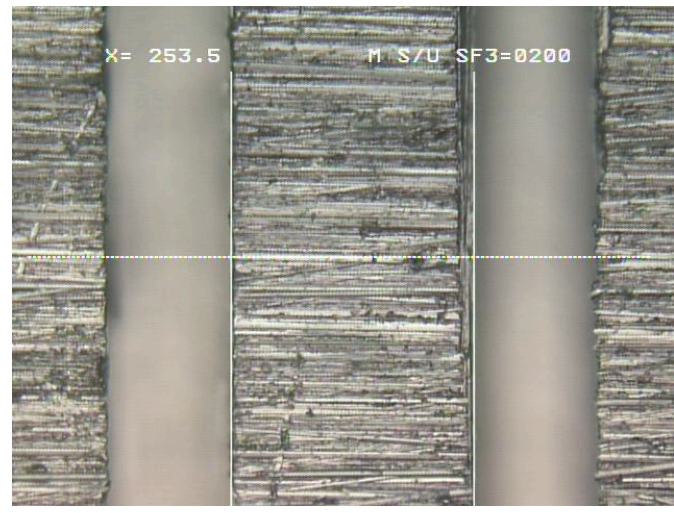

(D) Milled center span

FigURE 5.18 - Micrograph pictures of milled periodically notch PPW resonator. (A) Indicates the notches centered and milled across the width of the bottom plate of the NP-PPW, (B) a single notch width, (C) a single notch pitch, and (D) the center span length.

strength at a separation of $32 \mathrm{~mm}$. Then the waveguide was centered between the transmitted and receiver and fixed to the sample stage. The sample stage was then translated in the $x y$-plane until maximal signal strength was achieved.

FDTD simulations were performed using a broadband TM Gaussian source, as outlined in Section 3.2. Data for the transmitted field strength was collected by integrating the field component $H_{y}$ across the plate separation at the end of the PPW. Prior to collecting data for these experiments the FDTD simulation was verified using published results [24]. 


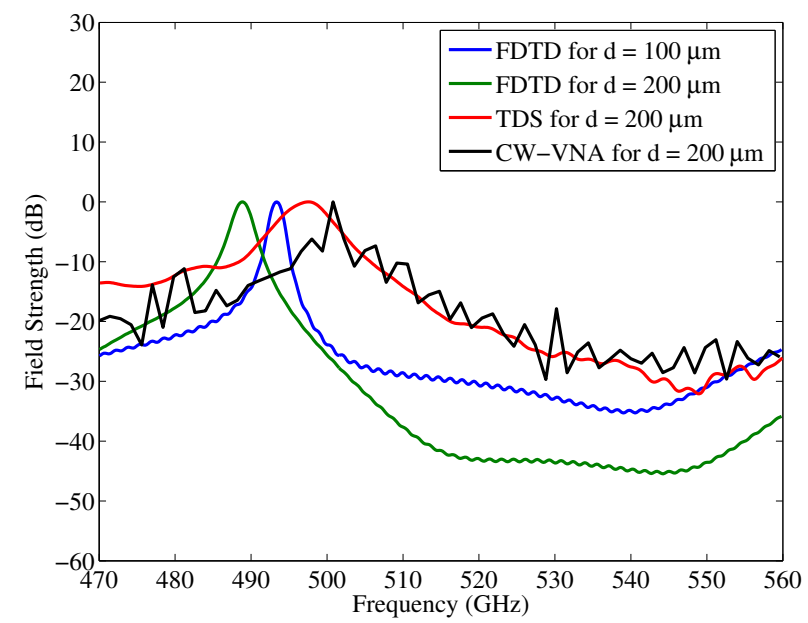

FiguRE 5.19 - Comparison of TDS and CW-VNA system results and FDTD simulated transmission characteristics of the NP-PPW resonator.

Results from TDS and CW-VNA systems are compared to FDTD simulations of varying plate separations in Figure 5.19. The resonant peak values for these results are summarized in Table 5.4. These results indicate that the resonant peak increases for smaller plate separations. This is shown in the difference in the resonant peaks of the TDS and CW-VNA systems. This discrepancy is attributed to imprecise measurements of the physical plate separation. In both experiments metal washers were used to maintain the plate separation. Then the top and bottom plates of the waveguide were screwed together by hand. Applying imprecise torque specifications creates inaccuracy in the actual plate separation value. This result is further ratified by the FDTD simulation results for $200 \mu \mathrm{m}$, where the resonant peak is lower than the TDS and CW-VNA results. This suggests that the actual plate separation was less than $200 \mu \mathrm{m}$. Although, the resonant peak of the FDTD simulation for $d=100 \mu \mathrm{m}$ still doesn't align with the measured values. The FDTD simulations indicate that halving plate separation corresponds to $\Delta \nu=4 \mathrm{GHz}$ and suggests a sensitivity of $\Delta \nu / \Delta d=40 \mathrm{MHz} / \mu \mathrm{m}$. Assuming a linear relationship between 


\begin{tabular}{|c|c|c|}
\hline & Resonance $(\mathbf{G H z})$ & $d(\mu \mathbf{m})$ \\
\hline TDS & 498 & 200 \\
\hline CW-VNA & 500 & 200 \\
\hline FDTD & 489 & 200 \\
\hline FDTD & 493 & 100 \\
\hline
\end{tabular}

TABLE 5.4 - TDS and CW-VNA system and FDTD simulation resonant peak values for varying plate separation.

the plate spacing and the resonant peak shift, aligning the simulations with the measured resonant peaks would not be possible. A possible explanation for this discrepancy is that the values used for $t, p, g$, and $w$ in the simulations differed from the physical PPW. While simulations affirm the behavior of the resonant peak shift wrt the plate separation spacing, it is unlikely that the exact plate separation can be ascertained from this set of simulations without more precise values for $t, p$, $g$, and $w$. These findings also suggest that the plate separation is less critical a design parameter for the resonant peak than the geometry and placement of notches. 


\section{Chapter 6}

\section{Conclusion and Future Work}

\subsection{Conclusion}

The region in the EM spectrum commonly known as the "THz gap" has attracted attention by the research community for its applications in spectroscopy, bio-medicine, and communications. While its presence has been known since the discovery of the EM spectrum, this region remained largely unexamined until technologies were developed to make efficient and compact THz sources and detectors. As such, the field of $\mathrm{THz}$ research is still considered in its infancy. As it matures, the focus of research in this region will move toward practical applications. The field of spectroscopy is a particularly intriguing area of $\mathrm{THz}$ radiation because many explosives and pharmaceuticals show unique spectral signatures in this region. The implications of a unique signature in this region of the EM spectrum suggests that $\mathrm{THz}$ radiation can be used to sense and identify materials. Additionally, by using the spectral signature the characteristic parameters of a material can be determined. The focus of this thesis was to characterize PPW multimode propagation using radiation patterns and investigate the effects of geometric changes to notched PPW resonant structures for use in practical sensing and material parameter extraction systems.

The spectral signature of a material is based on its ability to absorb only energy of specific wavelengths. When a source of broadband radiation is used to interrogate a material the subsequent energy that is not absorbed remains to be detected. It is this remaining detectible energy that makes the spectral signature 
of a material. Longer interactions with the penetrating radiation increase radiative absorption by the material. This ensures well defined absorption features in the spectra which allows a material's unique signature to be more easily identified. Without well defined absorption features, sensing systems would detect false positives and not detect false negatives. Additionally, without well defined absorption features material parameter extraction techniques would return unreliable results. This is particularly important in sensing applications where the amount of material is typically unknown. The PPW is a structure well suited for both sensing and material parameter extraction of small sample sizes. False readings can be diminished by designing PPW resonant structures that are tuned to detect only one material's unique spectral signature and by placing small sample sizes laterally inside the PPW the material-EM field interactions occur over longer distances than in free space. But, the use of PPW for broadband applications raises some interesting engineering challenges of its own. First, waveguide theory introduces multiple modes of operation which lead to excess energy loss in dispersion and mode conversion. By identifying and characterizing the behavior of the PPW operating in a multimode configuration, corrections may be applied to subsequent measurements, which would allow broadband multimode measurements to be performed. Second, resonance of the notched PPW structure is dependent on its geometric parameters; plate separation, notch width, depth, pitch, and center spacing. The plate separation can be adjusted (tuned) in-field, but the notch geometries and their placement must be determined prior to fabrication.

The results from this work demonstrate that PPW multimode operation is identifiable and that an estimate of the energy coupled into each mode is possible with radiation pattern measurements. The onset of the next higher order excited 
mode is identifiable in the striations of each respective field pattern spectrogram shown in Section 5.1.3. This is especially prevalent at large oblique angles for both the TE and TM mode. While the details of the spectrograms vary from TDS to FDTD to CW-VNA, they all show the same qualitative behavior. In all three cases it is easy to identify the onset of higher order modes at roughly the same frequencies.

Running simulations offers flexibility in PPW and source geometries for measurement comparison. The FDTD simulations include scattering effects, but unlike the analytic series solution, offer no control over the amount of multimode propagation. Therefore, FDTD simulations offer a more realistic glimpse into how a radiated multimode field should appear. Inclusion of scattering and interference effects in the FDTD simulation transfers energy into the radiation pattern side lobes and slightly shifts the angular position of the nulls. This effect distorts the distinct side lobes predicted by the analytic solution. Still, deviation from purely first order mode propagation is identifiable.

As shown in Section 5.1.4, the TDS measurements, the CW-VNA measurements and the FDTD simulations exhibit the same oscillatory behavior between well defined and undefined side lobe radiation patterns. Further, the exact frequencies in which undefined side lobes occur is inconsistent across the entire band for both systems and simulation. Taken separately, each result can be matched to a radiation pattern with well defined side lobes when the excess loss is at a minimum. Although, some overlap is observed across measurements and simulation. Despite this inconsistency, the fact that this behavior is observed for all indicates that it is not an artifact of the measurement systems or simulation but a physical phenomenon. The mechanism behind the oscillatory behavior of this excess loss and the distortion that is observed in the corresponding radiation patterns is not well 
understood at this point and merits further investigation. Although, disagreement across the experiments of the exact frequencies at which undefined side lobes occur is attributed to alignment mismatches in the measurement systems as compared to the exact geometries used for simulation.

For the same frequencies of interest, the TM dominant mode weighting coefficients are greater than the TE mode. This behavior is expected since the $\mathrm{TM}_{0}=\mathrm{TEM}$ mode is the first excited mode for an incident plane wave coupled into the PPW.

Results from N-PPW FDTD simulation indicate that relative to the case where $t / w=1$ the resonant peak increases for smaller depths and decreases for larger depths. Although, when the depth-to-width ratio $(t / w)$ is greater than 2 , there is no apparent shift in the resonant peak. For practical reasons the depth-towidth should be limited to less than 2 .

Cursory results for the NP-PPW indicate that the resonant peak increases for smaller plate separations. This is shown in the difference in the resonant peaks of the TDS and CW-VNA systems. FDTD simulation results show that halving the plate separation corresponds to $\Delta \nu=4 \mathrm{GHz}$ increase in the resonant peak. Assuming a linear relationship between the plate spacing and the resonant peak shift, suggests a sensitivity of $\Delta \nu / \Delta d=40 \mathrm{MHz} / \mu \mathrm{m}$. The discrepancy in the results between the systems is attributed to imprecise measurements of the physical plate separation. In both experiments metal washers were used to maintain the plate separation. Then the top and bottom plates of the waveguide were screwed together by hand. Applying imprecise torque specifications creates inaccuracy in the actual plate separation value. This result is further ratified by the FDTD simulation results for $200 \mu \mathrm{m}$, where the resonant peak is lower than the TDS and CW-VNA results. 
This suggests that the actual plate separation was less than $200 \mu \mathrm{m}$. Although, the resonant peak of the FDTD simulation for $d=100 \mu \mathrm{m}$ still doesn't align with the measured values. This discrepancy in simulation results suggest that the resonant peak is less dependent on plate separation than the geometry and placement of the notches. Despite this discrepancy, simulations do affirm the behavior of the resonant peak shift wrt the plate separation spacing.

\subsection{Future Work}

The very nature of research work is to push the limits of what is known and explore what is unknown. When we translate textbook theories to practical experiments or applications it seems inevitable that something is found that was not originally under investigation. This work is no exception and artifacts in the results which are not well understood at this time merit further investigation.

In the characterizing the PPW in multimode operation an oscillatory phenomenon was observed in which the side lobe radiation pattern predicted by the analytic solution was not seen observed. While this effect was verified in both measurement systems and through simulation, its mechanism of origin was not determined. It is worth noting that further work investigating the mechanisms contributing to this phenomenon is of interest in order to extend the modal coefficient estimation technique to the entire band. Initially, research into a geometric optic representation of the radiation pattern for a flanged PPW [31] should be considered. This type of theory would take into account the path length difference in the positive and negative transverse reflecting waves at the output aperture. This would investigate how scattering for frequencies whose last reflection were at or near the boundary of the aperture effect the radiation pattern. 
The BRMSD algorithm used to determine mode weighting coefficients merits further investigation. The algorithm doesn't take into consideration the input excitation of the PPW. The initial assumption that the dominant mode is excited first and higher order modes are excited in succession is based on a uniform plane wave input coupling. While this doesn't affect the final modal coefficient value, a more robust approach would take into consideration the input coupling excitation. This would reduce computation time and increase accuracy of the results by initially evaluating the propagating mode for the given excitation.

To reduce measurement times and improve accuracy in the radiation pattern characterization a fully automated measurement system should be developed. This would reduced the measurement alignment time and systematic errors in the process. Additionally, due to symmetry in the radiation patterns, with a more precise alignment only half the $\phi$-plane would need to be measured. This would also increase the accuracy of the modal weighting coefficients and decrease the computation time of the BRMSD algorithm, since only the half space would need to be evaluated.

The cursory investigation presented into PPW resonant structures opens up a number of interesting research opportunities. First, the mechanisms behind the diminishing returns wrt the notch depth-to-width ratio of the N-PPW should be investigated. The initial results indicate the resonant peak has a nonlinear dependence wrt the notch depth-to-width ratio. This relationship could be verified by further FDTD simulations for notch depth-to-width ratios of linear and logarithmic spacings. Second, the resonant peak for the NP-PPW FDTD simulations were not in agreement with the TDS and CW-VNA systems. This was attributed to differences in the simulated and physical resonant structure geometries. A logical validation of this hypothesis would be to rerun the FDTD simulations using the dimensional 
analysis values for the geometry of the resonant structure. The geometry of particular interest would be the center span dimension, since previous work by Nagel et. al [24] indicate that the resonant peak is determined by a center span dimension equal to $\lambda / 2$ of the resonant frequency. Finally, an analytic solution using the Fourier transform mode-matching technique for the NP-PPW would be of particular interest. Solutions for similar resonant geometries have been determined by Eom [8]. The NP-PPW geometry in question would be well suited for analysis by this technique due to its rectilinear geometry and periodic structures. 
REFERENCES

[1] R. Mendis and D. Mittleman, "An investigation of the lowest-order transverseelectric $\left(\mathrm{TE}_{1}\right)$ mode of the parallel-plate waveguide for $\mathrm{THz}$ pulse propagation," Optical Society of America, vol. 26, no. 9, pp. A6-A13, Sep 2009. (Cited on pages 4 and 47.)

[2] R. Mendis, "Thz waveguides: The evolution," in Infrared Millimeter Waves and 14th International Conference on Teraherz Electronics, 2006. IRMMW-THz 2006. Joint 31st International Conference on, sept. 2006, p. 367. (Cited on pages 4 and 48.)

[3] R. Mendis and D. Mittleman, "Comparision of the lowest-order transverseelectric $\left(\mathrm{TE}_{1}\right)$ and transverse-magnetic (TEM) modes of the parallel-plate waveguide for terahertz pulse applications," Optics Express, vol. 17, no. 17, pp. 14839-14850, Aug 2009. (Cited on pages 4, 48, and 60.)

[4] T. Park and H. Eom, "Analytic solution for TE-mode radiation from a flanged parallel-plate waveguide," IEE Proceedings- $H$, vol. 140 , no. 5, pp. 387-389, Oct 1993. (Cited on pages 5, 8, 9, 25, 29, 49, and 57.)

[5] A. F. Oskooi, D. Roundy, M. Ibanescu, P. Bermel, J. D. Joannopoulos, and S. G. Johnson, "MEEP: A flexible free-software package for electromagnetic simulations by the FDTD method," Computer Physics Communications, vol. 181, pp. 687-702, January 2010. (Cited on pages 5, 8, 9, 37, 49, and 58.) 
[6] D. Chien, K. Tanaka, and M. Tanaka, "Radiation properties of an arbitrarily flanged parallel-plate waveguide," IEEE Transactions on Antennas and Propagation, vol. 52, no. 8, pp. 1992-1999, Aug 2004. (Cited on pages 5 and 49.)

[7] H. J. Eom, Wave Scattering Theory - A Series Approach Based on the Fourier Transform. Springer, 2001. (Cited on pages 6, 25, 30, and 66.)

[8] —_, Electromagnetc Wave Theory for Boundary-Value Problems. Springer, 2004. (Cited on pages 6, 24, 25, 66, and 79.)

[9] G. Kniffin and L. Zurk, "Model-based material parameter estimation for terahertz reflection spectroscopy," Terahertz Science and Technology, IEEE Transactions on, vol. 2, no. 2, pp. $231-241$, march 2012. (Cited on page 7.)

[10] C. H. Kim, H. J. Eom, and T. J. Park, "A series for tm-mode radiation form a flanged parallel-plate waveguide," IEEE TRANSACTIONS ON ANTENNAS AND PROPAGATION AP, vol. 41, p. 1469, 1993. (Cited on pages 8, 9, 31, and 64.)

[11] A. Higgins, F. Kernan, and B. Pejcinovic, "Multimode characterization of parallel plate waveguide," in Microwave Measurement Symposium (ARFTG), 2010 76th ARFTG, 30 2010-dec. 3 2010, pp. 1 -5. (Cited on pages 8 and 9.)

[12] J. A. Higgins, F. Kernan, and B. Pejcinovic, "Application of open-source meep software to terahertz radiation waveguide problems," in MIPRO, 2011, pp. 118-123. (Cited on page 9.) 
[13] A. Higgins, B. Pejcinovic, C. Cowen, and F. Kernan, "An investigation of parallel plate waveguide terahertz radiation input coupling," SPIE Proceedings, vol. 8023, no. 1, pp. 802 310-802310-13, 2011. (Cited on pages 9 and 69.)

[14] F. Kernan, M. Wachsmuth, J. Higgins, B. Pejcinovic, L. Zurk, and R. Campbell, "Utilizing parallel plate waveguide modes for terahertz sensing," in Infrared Millimeter and Terahertz Waves (IRMMW-THz), 2010 35th International Conference on, sept. 2010, pp. $1-2$. (Cited on pages 9 and 10.)

[15] F. Kernan, A. Nadarajah, J. A. Higgins, B. Pejcinovic, and R. Koenenkamp, "Terahertz characterization of zinc oxide nanowires using parallel-plate waveguides," IEEE-NANO, vol. 11, 2011. (Cited on page 9.)

[16] J. C. Maxwell, A treatise on Electricity and Magnetism. Dover, N.Y., 1954. (Cited on page 11.)

[17] B. J. Hunt, The Maxwellians. Cornell University Press, 1991. (Cited on page 11.)

[18] D. J. Griffiths, Introduction to Electrodynamics, 3rd ed. Prentice Hall, 1999. (Cited on pages 12 and 21.)

[19] C. A. Balanis, Advanced Engineering Electromagnetics. Wiley, 1989. (Cited on pages 12 and 52.)

[20] D. M. Pozar, Microwave Engineering, 3rd ed. Wiley, 2005. (Cited on pages 12 and 21.)

[21] F. Ulaby, E. Michielssen, and U. Ravaioli, Fundamentals of Applied Electromagnetics, 6th ed. Prentice Hall, 2010. (Cited on page 12.) 
[22] L. Shen and J. Kong, Applied Electromagentism, 3rd ed. Cengage Learning, 2009. (Cited on page 12.)

[23] A. Taflove, Computational electrodynamics : the finite-difference time-domain method. Boston: Artech House, 1995. (Cited on page 32.)

[24] M. Nagel, P. H. Bolivar, and H. Kurz, "Modular parallel-plate thz components for cost-efficient biosensing systems," Semiconductor Science and Technology, vol. 20, no. 7, p. S281, 2005. (Cited on pages 38, 70, and 79.)

[25] T-Ray ${ }^{T M} 4000$ User Manual, Picometrix LLC, 2008. (Cited on page 42.)

[26] VDI Extender Module User Guide, Virginia Diodes Inc, 2009. (Cited on page 44.)

[27] M. Hiebel, "Vector network analyzer (vna) calibration: The basics," Rohde \& Schwarz, Tech. Rep., 2008. (Cited on page 50.)

[28] Vector Network Analyzers User Manual - ZVA, ZVB, ZVT, Rohde \& Schwarz - Test and Measurement Division. (Cited on page 50.)

[29] C. A. Balanis, Antenna Theory: Analysis and Design, 3rd ed. John Wiley \& Sons Inc., 2005. (Cited on page 52.)

[30] R. Mendis, V. Astley, J. Liu, and D. M. Mittleman, "Terahertz micofluidic sensor based on a parallel-plate waveguide resonant cavity," Applied Physics Letters, vol. 95, no. 171113, 2009. (Cited on page 67.)

[31] S. W. Lee, "Ray theory of diffraction by open-ended wageuides," Journal of Mathimatical Physics, vol. 11, no. 9, pp. 2830 - 2850, September 1970. (Cited on page 77 .) 